\title{
Littlest Seesaw
}

\section{Stephen F. King}

Physics and Astronomy, University of Southampton, Southampton, SO17 1BJ, U.K.

E-mail: king@soton.ac.uk

ABSTRACT: We propose the Littlest Seesaw (LS) model consisting of just two right-handed neutrinos, where one of them, dominantly responsible for the atmospheric neutrino mass, has couplings to $\left(\nu_{e}, \nu_{\mu}, \nu_{\tau}\right)$ proportional to $(0,1,1)$, while the subdominant right-handed neutrino, mainly responsible for the solar neutrino mass, has couplings to $\left(\nu_{e}, \nu_{\mu}, \nu_{\tau}\right)$ proportional to $(1, n, n-2)$. This constrained sequential dominance (CSD) model preserves the first column of the tri-bimaximal (TB) mixing matrix (TM1) and has a reactor angle $\theta_{13} \sim(n-1) \frac{\sqrt{2}}{3} \frac{m_{2}}{m_{3}}$. This is a generalisation of CSD $(n=1)$ which led to TB mixing and arises almost as easily if $n \geq 1$ is a real number. We derive exact analytic formulas for the neutrino masses, lepton mixing angles and CP phases in terms of the four input parameters and discuss exact sum rules. We show how CSD $(n=3)$ may arise from vacuum alignment due to residual symmetries of $S_{4}$. We propose a benchmark model based on $S_{4} \times Z_{3} \times Z_{3}^{\prime}$, which fixes $n=3$ and the leptogenesis phase $\eta=2 \pi / 3$, leaving only two inputs $m_{a}$ and $m_{b}=m_{e e}$ describing $\Delta m_{31}^{2}, \Delta m_{21}^{2}$ and $U_{\text {PMNS. }}$. The LS model predicts a normal mass hierarchy with a massless neutrino $m_{1}=0$ and TM1 atmospheric sum rules. The benchmark LS model additionally predicts: solar angle $\theta_{12}=34^{\circ}$, reactor angle $\theta_{13}=8.7^{\circ}$, atmospheric angle $\theta_{23}=46^{\circ}$, and Dirac phase $\delta_{\mathrm{CP}}=-87^{\circ}$.

Keywords: Neutrino Physics, Discrete Symmetries, Solar and Atmospheric Neutrinos

ARXiv EPrint: 1512.07531 
Contents

1 Introduction 2

2 Seesaw mechanism with two right-handed neutrinos 4

$\begin{array}{llr}3 & \text { Trimaximal mixing } & 6\end{array}$

4 Flavour symmetry: direct versus indirect models $\quad 7$

5 Indirect approach and vacuum alignment $\quad 8$

6 The Littlest Seesaw $\quad 9$

7 A numerical benchmark: $\operatorname{CSD}(3)$ with $\eta=2 \pi / 3 \quad 10$

8 Exact analytic results for lepton mixing angles 11

9 Exact analytic results for neutrino masses $\quad 14$

10 Exact analytic results for CP violation $\quad \mathbf{1 5}$

$\begin{array}{ll}10.1 \text { The Jarlskog invariant } & 15\end{array}$

$\begin{array}{ll}10.2 \text { The Majorana invariant } & 17\end{array}$

11 Exact sum rules $\quad 18$

12 The reactor and atmospheric angles $\quad 19$

$\begin{array}{ll}12.1 \text { The reactor angle } & 20\end{array}$

$\begin{array}{ll}12.2 \text { The atmospheric angle } & 21\end{array}$

$13 \operatorname{CSD}(3)$ vacuum alignments from $S_{4} \quad 22$

14 A benchmark model with $S_{4} \times Z_{3} \times Z_{3}^{\prime} \quad 23$

15 Vacuum alignment in the $S_{4} \times Z_{3} \times Z_{3}^{\prime}$ model 26

$\begin{array}{lr}16 \text { Conclusion } & 28\end{array}$

$\begin{array}{lr}\text { A Lepton mixing conventions } & 29\end{array}$

$\begin{array}{ll}\text { B } S_{4} & 30\end{array}$ 


\section{Introduction}

The discovery of neutrino oscillations, implying mass and mixing, remains one of the greatest discoveries in physics in the last two decades. Although the origin of neutrino mass is presently unknown (for reviews see e.g. [1-4]), whatever is responsible must be new physics beyond the Standard Model (BSM). For example, the leading candidate for neutrino mass and mixing is the seesaw mechanism involving additional right-handed neutrinos with heavy Majorana masses [5-10], providing an elegant explanation of the smallness of neutrino mass. ${ }^{1}$

However, in general, the seesaw mechanism typically involves many parameters, making quantitative predictions of neutrino mass and mixing challenging. In this respect, the seesaw mechanism offers no more understanding of flavour than the Yukawa couplings of the SM. Indeed it introduces a new flavour sector associated with right-handed neutrino Majorana masses, which cannot be directly probed by high energy particle physics experiment. Clearly a different approach is required to make progress with the new (or nu) Standard Model that involves the seesaw mechanism. Here we shall make use of the theoretical touchstones of elegance and simplicity (which indeed motivate the seesaw mechanism in the first place) to try to allow some experimental guidance to inform the high energy seesaw mechanism. If the assumptions we make prove to be inconsistent with experiment then we must think again, otherwise the framework of assumptions remains viable.

In this paper, then, we focus on natural implementations of the seesaw mechanism, where typically one of the right-handed neutrinos is dominantly responsible for the atmospheric neutrino mass $[12,13]$, while a second subdominant right-handed neutrino accounts for the solar neutrino mass [14]. This idea of sequential dominance (SD) of right-handed neutrinos is an elegant hypothesis which, when combined with the assumption of a zero coupling of the atmospheric neutrino to $\nu_{e}$, leads to the generic bound $\theta_{13} \lesssim m_{2} / m_{3}[15,16]$, which appears to be approximately saturated according to current measurements of the reactor angle. This bound was derived over a decade before the experimental measurement of the reactor angle. This success supports the SD approach, and motivates efforts to understand why the reactor bound is approximately saturated. In order to do this one needs to further constrain the Yukawa couplings beyond the assumption of a single texture zero as assumed above.

The idea of constrained sequential dominance (CSD) is that the "atmospheric" righthanded neutrino has couplings to $\left(\nu_{e}, \nu_{\mu}, \nu_{\tau}\right)$ proportional to $(0,1,1)$, while the "solar" right-handed neutrino has couplings to $\left(\nu_{e}, \nu_{\mu}, \nu_{\tau}\right)$ proportional to $(1, n, n-2)$ where $n$ is a real number. It turns out that such a structure preserves the first column of the tri-bimaximal (TB) [17] mixing matrix (TM1), leading to the approximate result $\theta_{13} \sim$ $(n-1) \frac{\sqrt{2}}{3} \frac{m_{2}}{m_{3}}$, which we shall derive here from exact results. This scheme is therefore a generalisation of the original $\operatorname{CSD}(n=1)$ [18] which led to TB mixing and the prediction $\theta_{13}=0$, which is now excluded. It is also a generalisation of $\operatorname{CSD}(n=2)$ [19] which predicted $\theta_{13} \sim \frac{\sqrt{2}}{3} \frac{m_{2}}{m_{3}}$, which was subsequently proved to be too small when more precise measurements of $\theta_{13}$ were made. It seems we are third time lucky since $\operatorname{CSD}(n=3)$ [20]

\footnotetext{
${ }^{1}$ For a simple introduction to the seesaw mechanism see e.g. [11].
} 
predicts $\theta_{13} \sim 2 \frac{\sqrt{2}}{3} \frac{m_{2}}{m_{3}}$ which is in broad agreement with current measurements. On the other hand $\operatorname{CSD}(n=4)[21,22]$ predicts $\theta_{13} \sim \sqrt{2} \frac{m_{2}}{m_{3}}$ appears to be a little too large, unless a third right-handed neutrino is invoked (unavoidable in Pati-Salam for example [23]), while $\operatorname{CSD}(n \geq 5)$ [24] is excluded. Recently, $\operatorname{CSD}(3)$ has been exploited in Grand Unified Theories (GUTs) based on SU(5) [25, 26] and $\mathrm{SO}(10)$ [27].

The new approach and results in this paper are summarised below:

- The approach here is more general than previously considered, since we allow $n$ to be a real number, rather than being restricted to the field of positive integers. The motivation is that the vacuum alignment vector $(1, n, n-2)$ is orthogonal to the first column of the TB matrix $(2,-1,1)$ (which in turn is orthogonal to the second and third TB columns $(1,1,-1)$ and $(0,1,1))$ for any real number $n$, emerges very naturally as depicted in figure 1 . This provides a plausible motivation for considering the vacuum alignment direction $(1, n, n-2)$ for any real number $n$. We refer to the associated minimal models as the Littlest Seesaw (LS). The LS with $\operatorname{CSD}(n)$ predicts a normal mass hierarchy with a massless neutrino $m_{1}=0$, both testable in the near future. Actually the above predictions also arise in general two right-handed neutrino models. What distinguishes the LS model from general two right-handed neutrino models are the predictions for the lepton mixing angles and $\mathrm{CP}$ phases as discussed below.

- For the general case of any real value of $n \geq 1$, for the first time we shall derive exact analytic formulas for the neutrino masses, lepton mixing angles and CP phases (both Dirac and Majorana) in terms of the four input parameters. This is progress since previously only numerical results were used. We also show that $\operatorname{CSD}(n)$ is subject to the TM1 mixing sum rules and no other ones. From the exact results, which are useful for many purposes but a little lengthy, we extract some simple approximations which provide some rough and ready insight into what is going on. For example, the approximate result $\theta_{13} \sim(n-1) \frac{\sqrt{2}}{3} \frac{m_{2}}{m_{3}}$ provides an analytic understanding of why $\operatorname{CSD}(n \geq 5)$ is excluded, which until now has only been a numerical finding.

- We show that the successful case of $\operatorname{CSD}(3)$ arises more naturally from symmetry in the case of $S_{4}$, rather than using $A_{4}$, as was done in previous work [20-25]. The reason is that both the neutrino scalar vacuum alignments $(0,1,1)$ and $(1,3,1)$ preserve residual subgroups of $S_{4}$ which are not present in $A_{4}$. This motivates models based on $S_{4}$, extending the idea of residual symmetries from the confines of two sectors (the charged lepton and neutrino sectors) as is traditionally done in direct models, to five sectors, two associated with the neutrinos and three with the charged leptons, as summarised in the starfish shaped diagram in figure 2 .

- Finally we present a benchmark LS model based on $S_{4} \times Z_{3} \times Z_{3}^{\prime}$, with supersymmetric vacuum alignment, which not only fixes $n=3$ but also the leptogenesis phase $\eta=$ $2 \pi / 3$, leaving only two continuous input masses, yielding two neutrino mass squared splittings and the PMNS matrix. A single $Z_{3}$ factor is required to understand $\eta=$ 
$2 \pi / 3$ as a cube root of unity, while an additional $Z_{3}^{\prime}$ is necessary to understand the charged lepton mass hierarchy and also to help to control the operator structure of the model. The model provides a simple LS framework for the numerical benchmark predictions: solar angle $\theta_{12}=34^{\circ}$, reactor angle $\theta_{13}=8.7^{\circ}$, atmospheric angle $\theta_{23}=$ $46^{\circ}$, and Dirac phase $\delta_{\mathrm{CP}}=-87^{\circ}$, which are readily testable in forthcoming oscillation experiments.

The layout of the remainder of the paper is as follows. In section 2 we briefly introduce the two right-handed neutrino model and motivate $\operatorname{CSD}(n)$. In section 3 we show how $\operatorname{CSD}(n)$ implies TM1 mixing. In section 4 we briefly review the direct and indirect approaches to model building, based on flavour symmetry. In section 5 we pursue the indirect approach and show how vacuum alignment for $\operatorname{CSD}(n)$ can readily be obtained from the TB vacuum alignments using orthogonality. In section 6 we write down the Lagrangian of the LS model and derive the neutrino mass matrix from the seesaw mechanism with $\operatorname{CSD}(n)$. In section 7 we discuss a numerical benchmark, namely $\operatorname{CSD}(3)$ with leptogenesis phase $\eta=2 \pi / 3$ and its connection with the oscillation phase. In sections 8, 9, 10 we derive exact analytic formulas for the angles, masses and CP phases, for the LS model with general $\operatorname{CSD}(n)$ valid for real $n \geq 1$, in terms of the four input parameters of the model. In section 11 present the exact TM1 atmospheric sum rules, which we argue are the only ones satisfied by the model. In section 12 we focus on the reactor and atmospheric angles and, starting from the exact results, derive useful approximate formulae which can provide useful insight. In section 13 we show how vacuum alignment for $\operatorname{CSD}(3)$ can arise from the residual symmetries of $S_{4}$, as summarised by the starfish diagram in figure 2 . In sections 14 and 15 we present a benchmark LS model based on the discrete group $S_{4} \times Z_{3} \times Z_{3}^{\prime}$, with supersymmetric vacuum alignment, which not only fixes $n=3$ but also the leptogenesis phase $\eta=2 \pi / 3$, reproducing the parameters of the numerical benchmark. Section 16 concludes the paper. There are two appendices, appendix A on lepton mixing conventions and appendix B on $S_{4}$.

\section{Seesaw mechanism with two right-handed neutrinos}

The two right-handed neutrino seesaw model was first proposed in [14]. Subsequently two right-handed neutrino models with two texture zeros were discussed in [28], however such two texture zero models are now phenomenologically excluded [29] for the case of a normal neutrino mass hierarchy considered here. However the two right-handed neutrino model with one texture zero (actually also suggested in [14]), remains viable.

With two right-handed neutrinos, the Dirac mass matrix $m^{D}$ is, in LR convention,

$$
m^{D}=\left(\begin{array}{ll}
d & a \\
e & b \\
f & c
\end{array}\right), \quad\left(m^{D}\right)^{T}=\left(\begin{array}{lll}
d & e & f \\
a & b & c
\end{array}\right)
$$

The (diagonal) right-handed neutrino heavy Majorana mass matrix $M_{R}$ with rows 
$\left(\overline{\nu_{R}^{\mathrm{atm}}}, \overline{\nu_{R}^{\mathrm{sol}}}\right)^{T}$ and columns $\left(\nu_{R}^{\mathrm{atm}}, \nu_{R}^{\mathrm{sol}}\right)$ is,

$$
M_{R}=\left(\begin{array}{cc}
M_{\mathrm{atm}} & 0 \\
0 & M_{\mathrm{sol}}
\end{array}\right), \quad M_{R}^{-1}=\left(\begin{array}{cc}
M_{\mathrm{atm}}^{-1} & 0 \\
0 & M_{\mathrm{sol}}^{-1}
\end{array}\right)
$$

The light effective left-handed Majorana neutrino mass matrix is given by the seesaw formula

$$
m^{\nu}=-m^{D} M_{R}^{-1} m^{D^{T}},
$$

Using the see-saw formula dropping the overall minus sign which is physically irrelevant, we find, by multiplying the matrices in eqs. (2.1), (2.2),

$$
m^{\nu}=m^{D} M_{R}^{-1}\left(m^{D}\right)^{T}=\left(\begin{array}{lll}
\frac{a^{2}}{M_{\mathrm{sol}}}+\frac{d^{2}}{M_{\mathrm{atm}}} \frac{a b}{M_{\mathrm{sol}}}+\frac{d e}{M_{\mathrm{atm}}} \frac{a c}{M_{\mathrm{sol}}}+\frac{d f}{M_{\mathrm{atm}}} \\
\frac{a b}{M_{\mathrm{sol}}}+\frac{d e}{M_{\mathrm{atm}}} \frac{b^{2}}{M_{\mathrm{sol}}}+\frac{e^{2}}{M_{\mathrm{atm}}} \frac{b c}{M_{\mathrm{sol}}}+\frac{e f}{M_{\mathrm{atm}}} \\
\frac{a c}{M_{\mathrm{sol}}}+\frac{d f}{M_{\mathrm{atm}}} \frac{b c}{M_{\mathrm{sol}}}+\frac{e f}{M_{\mathrm{atm}}} \frac{c^{2}}{M_{\mathrm{sol}}}+\frac{f^{2}}{M_{\mathrm{atm}}}
\end{array}\right)
$$

Motivated by the desire to implement the seesaw mechanism in a natural way, sequential dominance (SD) [12-14] assumes that the two right-handed neutrinos $\nu_{R}^{\text {sol }}$ and $\nu_{R}^{\text {atm }}$ have couplings $d \ll e, f$ and

$$
\frac{(e, f)^{2}}{M_{\mathrm{atm}}} \gg \frac{(a, b, c)^{2}}{M_{\mathrm{sol}}} .
$$

By explicit calculation, using eq. (2.4), one can check that in the two right-handed neutrino limit $\operatorname{det} m^{\nu}=0$. Since the determinant of a Hermitian matrix is the product of mass eigenvalues

$$
\operatorname{det}\left(m^{\nu} m^{\nu \dagger}\right)=m_{1}^{2} m_{2}^{2} m_{3}^{2},
$$

one may deduce that one of the mass eigenvalues of the complex symmetric matrix above is zero, which under the SD assumption is the lightest one $m_{1}=0$ with $m_{3} \gg m_{2}$ since the model approximates to a single right-handed neutrino model $[12,13]$. Hence we see that SD implies a normal neutrino mass hierarchy. Including the solar right-handed neutrino as a perturbation, it can be shown that, for $d=0$, together with the assumption of a dominant atmospheric right-handed neutrino in eq. (2.5), leads to the approximate results for the solar and atmospheric angles [12-14],

$$
\tan \theta_{23} \sim \frac{e}{f}, \quad \tan \theta_{12} \sim \frac{\sqrt{2} a}{b-c} .
$$

Under the above SD assumption, each of the right-handed neutrinos contributes uniquely to a particular physical neutrino mass. The SD framework above with $d=0$ leads to the relations in eq. (2.6) together with the reactor angle bound [15, 16],

$$
\theta_{13} \lesssim \frac{m_{2}}{m_{3}}
$$

This result shows that SD allows for large values of the reactor angle, consistent with the measured value. Indeed the measured reactor angle, observed a decade after this theoretical bound was derived, approximately saturates the upper limit. In order to understand why this is so, we must go beyond the SD assumptions stated so far. 
Motivated by the desire to obtain an approximately maximal atmospheric angle $\tan \theta_{23} \sim 1$ and trimaximal solar angle $\tan \theta_{12} \sim 1 / \sqrt{2}$, the results in eq. (2.6) suggest constraining the Dirac matrix elements in eq. (2.1) to take the values $d=0$ with $e=f$ and $b=n a$ and also $c=(n-2) a$,

$$
m^{D}=\left(\begin{array}{cc}
0 & a \\
e & n a \\
e & (n-2) a
\end{array}\right),
$$

which, for any positive integer $n$, is referred to as constrained sequential dominance (CSD) [18-24]. In section 12 we shall show that eq. (2.8) also implies,

$$
\theta_{13} \sim(n-1) \frac{\sqrt{2}}{3} \frac{m_{2}}{m_{3}}
$$

so that the bound in eq. (2.7) is approximately saturated for $n \sim 3$.

As already mentioned, we refer to a two right-handed neutrino model in which the Dirac mass matrix in the flavour basis satisfies eq. (2.8), with $n$ being a real number, as the "Littlest Seesaw" or LS model. The justification for this terminology is that it represents the seesaw model with the fewest number of parameters consistent with current neutrino data. To be precise, in the flavour basis, the Dirac mass matrix of the LS model involves two complex parameters $e, a$ plus one real parameter $n$. This is fewer than the original two right-handed neutrino Dirac mass matrix which involves six complex parameters [14]. It is also fewer than the two right-handed neutrino model in $[15,16]$ which involves five complex parameters due to the single texture zero. It is even fewer than the minimal right-handed neutrino model in [28] which involves four complex parameters due to the two texture zeroes. It remains to justify the Dirac structure of the LS model in eq. (2.8), and we shall address this question using symmetry and vacuum alignment in subsequent sections.

\section{Trimaximal mixing}

A simple example of lepton mixing which came to dominate the model building community until the measurement of the reactor angle is the tribimaximal (TB) mixing matrix [17]. It predicts zero reactor angle $\theta_{13}=0$, maximal atmospheric angle $s_{23}^{2}=1 / 2$, or $\theta_{12}=45^{\circ}$, and a solar mixing angle given by $s_{12}=1 / \sqrt{3}$, i.e. $\theta_{12} \approx 35.3^{\circ}$. The mixing matrix is given explicitly by

$$
U_{\mathrm{TB}}=\left(\begin{array}{ccc}
\sqrt{\frac{2}{3}} & \frac{1}{\sqrt{3}} & 0 \\
-\frac{1}{\sqrt{6}} & \frac{1}{\sqrt{3}} & \frac{1}{\sqrt{2}} \\
\frac{1}{\sqrt{6}} & -\frac{1}{\sqrt{3}} & \frac{1}{\sqrt{2}}
\end{array}\right) .
$$

Unfortunately TB mixing is excluded since it predicts a zero reactor angle. However CSD in eq. (2.8) with two right-handed neutrinos allows a non-zero reactor angle for $n>1$ and also predicts the lightest physical neutrino mass to be zero, $m_{1}=0$. One can also check that the neutrino mass matrix resulting from using eq. (2.8) in the seesaw formula 
in eq. (2.3), satisfies

$$
m^{\nu}\left(\begin{array}{c}
2 \\
-1 \\
1
\end{array}\right)=\left(\begin{array}{l}
0 \\
0 \\
0
\end{array}\right) .
$$

In other words the column vector $(2,-1,1)^{T}$ is an eigenvector of $m^{\nu}$ with a zero eigenvalue, i.e. it is the first column of the PMNS mixing matrix, corresponding to $m_{1}=0$, which means so called TM1 mixing [30-32] in which the first column of the TB mixing matrix in eq. (3.1) is preserved, while the other two columns are allowed to differ (in particular the reactor angle will be non-zero for $n>1$ ),

$$
U_{\mathrm{TM} 1}=\left(\begin{array}{cc}
\sqrt{\frac{2}{3}} & -- \\
-\frac{1}{\sqrt{6}} & -- \\
\frac{1}{\sqrt{6}} & --
\end{array}\right) .
$$

Interestingly CSD in eq. (2.8) with $n=1$ [18] predicts a zero reactor angle and hence TB mixing, while for $n>1$ it simply predicts the less restrictive TM1 mixing. Having seen that CSD leads to TB, or more generally TM1 mixing, we now discuss the theoretical origin of the desired Dirac mass matrix structure in eq. (2.8).

\section{Flavour symmetry: direct versus indirect models}

Let us expand the neutrino mass matrix in the diagonal charged lepton basis, assuming exact TB mixing, as $m_{T B}^{\nu}=U_{T B} \operatorname{diag}\left(m_{1}, m_{2}, m_{3}\right) U_{T B}^{T}$ leading to (absorbing the Majorana phases in $\left.m_{i}\right)$ :

$$
m_{T B}^{\nu}=m_{1} \Phi_{1} \Phi_{1}^{T}+m_{2} \Phi_{2} \Phi_{2}^{T}+m_{3} \Phi_{3} \Phi_{3}^{T}
$$

where

$$
\Phi_{1}^{T}=\frac{1}{\sqrt{6}}(2,-1,1), \quad \Phi_{2}^{T}=\frac{1}{\sqrt{3}}(1,1,-1), \quad \Phi_{3}^{T}=\frac{1}{\sqrt{2}}(0,1,1),
$$

are the respective columns of $U_{T B}$ and $m_{i}$ are the physical neutrino masses. In the neutrino flavour basis (i.e. diagonal charged lepton mass basis), it has been shown that the above TB neutrino mass matrix is invariant under $S, U$ transformations:

$$
m_{T B}^{\nu}=S m_{T B}^{\nu} S^{T}=U m_{T B}^{\nu} U^{T} .
$$

A very straightforward argument [33] shows that this neutrino flavour symmetry group has only four elements corresponding to Klein's four-group $Z_{2}^{S} \times Z_{2}^{U}$. By contrast the diagonal charged lepton mass matrix (in this basis) satisfies a diagonal phase symmetry $T$. In the case of TB mixing, the matrices $S, T, U$ form the generators of the group $S_{4}$ in the triplet representation, while the $A_{4}$ subgroup is generated by $S, T$.

As discussed in [33], the flavour symmetry of the neutrino mass matrix may originate from two quite distinct classes of models. The class of models, which we call direct models, are based on a family symmetry such as $S_{4}$, for example, where the symmetry of the neutrino mass matrix is a remnant of the $S_{4}$ symmetry of the Lagrangian, with the generators $S, U$ preserved in the neutrino sector, while the diagonal generator $T$ is preserved 
in the charged lepton sector. If $U$ is broken but $S$ is preserved, then this leads to TM2 mixing with the second column of the TB mixing matrix being preserved. However if the combination $S U$ is preserved then this corresponds to TM1 mixing with the first column of the TB mixing matrix being preserved [34]. Of course, the $S_{4}$ symmetry is completely broken in the full lepton Lagrangian including both neutrino and charged lepton sectors.

In an alternative class of models, which we call indirect models, the family symmetry is already completely broken in the neutrino sector, where the observed neutrino flavour symmetry $Z_{2}^{S} \times Z_{2}^{U}$ emerges as an accidental symmetry. However the structure of the Dirac mass matrix is controlled by vacuum alignment in the flavour symmetry breaking sector, as discussed in the next section. The indirect models are arguably more natural than the direct models, especially for $m_{1}=0$, since each column of the Dirac mass matrix corresponds to a different symmetry breaking VEV and each contribution to the seesaw mechanism corresponds to a different right-handed neutrino mass, enabling mass hierarchies to naturally emerge. Thus a strong mass hierarchy $m_{1} \ll m_{2}<m_{3}$ would seem to favour indirect models over direct models, so we pursue this possibility in the following.

\section{Indirect approach and vacuum alignment}

The basic idea of the indirect approach is to effectively promote the columns of the Dirac mass matrix to fields which transform as triplets under the flavour symmetry. We assume that the Dirac mass matrix can be written as $m_{D}=\left(a \Phi_{\mathrm{atm}}, b \Phi_{\mathrm{sol}}, c \Phi_{\mathrm{dec}}\right)$ where the columns are proportional to triplet Higgs scalar fields with particular vacuum alignments and $a, b, c$ are three constants of proportionality. Working in the diagonal right-handed neutrino mass basis, the seesaw formula gives,

$$
m^{\nu}=a^{2} \frac{\Phi_{\mathrm{atm}} \Phi_{\mathrm{atm}}^{T}}{M_{\mathrm{atm}}}+b^{2} \frac{\Phi_{\mathrm{sol}} \Phi_{\mathrm{sol}}^{T}}{M_{\mathrm{sol}}}+c^{2} \frac{\Phi_{\mathrm{dec}} \Phi_{\mathrm{dec}}^{T}}{M_{\mathrm{dec}}},
$$

By comparing eq. (5.1) to the TB form in eq. (4.1) it is clear that TB mixing will be achieved if $\Phi_{\mathrm{atm}} \propto \Phi_{3}$ and $\Phi_{\mathrm{sol}} \propto \Phi_{2}$ and $\Phi_{\mathrm{dec}} \propto \Phi_{1}$, with each of $m_{3,2,1}$ originating from a particular right-handed neutrino. The case where the columns of the Dirac mass matrix are proportional to the columns of the PMNS matrix, the columns being therefore mutually orthogonal, is referred to as form dominance (FD) [35-37]. The resulting $m^{\nu}$ is form diagonalizable. Each column of the Dirac mass matrix arises from a separate flavon VEV, so the mechanism is very natural, especially for the case of a strong mass hierarchy. Note that for $m_{1} \ll m_{2}<m_{3}$ the precise form of $\Phi_{\text {dec }}$ becomes irrelevant and for $m_{1}=0$ we can simply drop the last term and the model reduces to a two right-handed neutrino model.

Within this framework, the general CSD Dirac mass matrix structure in eq. (2.8) corresponds to there being some Higgs triplets which can be aligned in the directions,

$$
\Phi_{\mathrm{atm}}^{T} \propto(0,1,1), \quad \Phi_{\mathrm{sol}}^{T} \propto(1, n, n-2),
$$

The first vacuum alignment $\Phi_{\text {atm }}$ in eq. (5.2) is just the TB direction $\Phi_{3}^{T}$ in eq. (4.2). The second vacuum alignment $\Phi_{\text {sol }}$ in eq. (5.2) can be easily obtained since the direction $(1, n, n-2)$ is orthogonal to the TB vacuum alignment $\Phi_{1}^{T}$ in eq. (4.2). 


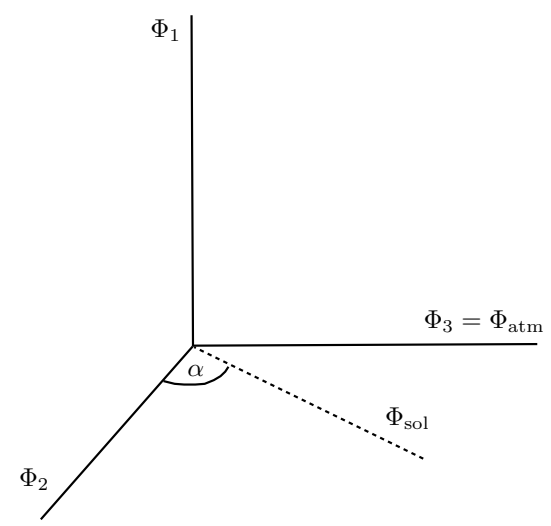

Figure 1. The mutually orthogonal vacuum alignments $\Phi_{i}$ in eq. (4.2) used for TB mixing. The alignment vector $\Phi_{\text {sol }}$ is orthogonal to $\Phi_{1}$ and hence is in the plane defined by $\Phi_{2}$ and $\Phi_{3}=\Phi_{\text {atm }}$. Note that the vectors $\Phi_{\text {sol }}$ and $\Phi_{\text {atm }}$ in eq. (5.2) are not orthogonal for a general value of $n$, so any seesaw model based on these alignments will violate form dominance.

For example, in a supersymmetric theory, the aligning superpotential should contain the following terms, enforced by suitable discrete $Z_{n}$ symmetries,

$$
O_{i j} \phi_{i} \phi_{j}+O_{\mathrm{sol}} \phi_{\mathrm{sol}} \phi_{1}
$$

where the terms proportional to the singlets $O_{i j}$ and $O_{\text {sol }}$ ensure that the real $S_{4}$ triplets are aligned in mutually orthogonal directions, $\Phi_{i} \perp \Phi_{j}$ and $\Phi_{\text {sol }} \perp \Phi_{1}$ as depicted in figure 1 . From eqs. (4.2), (5.2) we can write $\Phi_{\text {sol }}=\Phi_{2} \cos \alpha+\Phi_{3} \sin \alpha$ where $\tan \alpha=2(n-1) / 3$ and $\alpha$ is the angle between $\Phi_{\text {sol }}$ and $\Phi_{2}$, as shown in figure 1. $\Phi_{\text {sol }}$ is parallel to $\Phi_{2}$ for $n=1$, while it increasingly tends towards the $\Phi_{3}=\Phi_{\text {atm }}$ alignment as $n$ is increased.

\section{The Littlest Seesaw}

The littlest seesaw (LS) model consists of the three families of electroweak lepton doublets $L$ unified into a single triplet of the flavour symmetry, while the two right-handed neutrinos $\nu_{R}^{\mathrm{atm}}$ and $\nu_{R}^{\mathrm{sol}}$ are singlets. The LS Lagrangian in the neutrino sector takes the form,

$$
\mathcal{L}=-y_{\mathrm{atm}} \bar{L} \cdot \phi_{\mathrm{atm}} \nu_{R}^{\mathrm{atm}}-y_{\mathrm{sol}} \bar{L} \cdot \phi_{\mathrm{sol}} \nu_{R}^{\mathrm{sol}}-\frac{1}{2} M_{\mathrm{atm}} \bar{\nu}_{R}^{\mathrm{c}}{ }_{\mathrm{atm}}^{\mathrm{atm}}-\frac{1}{2} M_{\mathrm{sol}} \bar{\nu}_{R}^{\mathrm{c}} \mathrm{sol} \nu_{R}^{\mathrm{sol}}+H . c . .
$$

which may be enforced by suitable discrete $Z_{3}$ symmetries, as discussed in section 14 . Here $\phi_{\text {sol }}$ and $\phi_{\text {atm }}$ may be interpreted as either Higgs fields, which transform as triplets of the flavour symmetry with the alignments in eq. (5.2), or as combinations of a single Higgs electroweak doublet together with triplet flavons with these vacuum alignments. Note that, in eq. (6.1), $\phi_{\text {sol }}$ and $\phi_{\text {atm }}$ represent fields, whereas in eq. (5.2) $\Phi_{\text {sol }}$ and $\Phi_{\text {atm }}$ refer to the VEVs of those fields.

In the diagonal charged lepton and right-handed neutrino mass basis, when the fields $\Phi_{\text {sol }}$ and $\Phi_{\text {atm }}$ in eq. (6.1) are replaced by their VEVs in eq. (5.2), this reproduces the Dirac 
mass matrix in eq. (2.8) [20] and its transpose:

$$
m^{D}=\left(\begin{array}{cc}
0 & a \\
e & n a \\
e & (n-2) a
\end{array}\right), \quad m^{D^{T}}=\left(\begin{array}{ccc}
0 & e & e \\
a & n a & (n-2) a
\end{array}\right),
$$

which defines the LS model, where we regard $n$ as a real continuous parameter, later arguing that it may take simple integer values. The (diagonal) right-handed neutrino heavy Majorana mass matrix $M_{R}$ with rows $\left(\bar{\nu}_{R}^{\text {atm }}, \nu_{R}^{c} \text { sol }\right)^{T}$ and columns $\left(\nu_{R}^{\text {atm }}, \nu_{R}^{\text {sol }}\right)$ is,

$$
M_{R}=\left(\begin{array}{cc}
M_{\mathrm{atm}} & 0 \\
0 & M_{\mathrm{sol}}
\end{array}\right), \quad M_{R}^{-1}=\left(\begin{array}{cc}
M_{\mathrm{atm}}^{-1} & 0 \\
0 & M_{\mathrm{sol}}^{-1}
\end{array}\right)
$$

The low energy effective Majorana neutrino mass matrix is given by the seesaw formula

$$
m^{\nu}=-m^{D} M_{R}^{-1} m^{D^{T}},
$$

which, after multiplying the matrices in eqs. (6.2), (6.3), for a suitable choice of physically irrelevant overall phase, gives

$$
m^{\nu}=m_{a}\left(\begin{array}{lll}
0 & 0 & 0 \\
0 & 1 & 1 \\
0 & 1 & 1
\end{array}\right)+m_{b} e^{i \eta}\left(\begin{array}{ccc}
1 & n & (n-2) \\
n & n^{2} & n(n-2) \\
(n-2) & n(n-2) & (n-2)^{2}
\end{array}\right),
$$

where $\eta$ is the only physically important phase, which depends on the relative phase between the first and second column of the Dirac mass matrix, $\arg (a / e)$ and $m_{a}=\frac{|e|^{2}}{M_{\text {atm }}}$ and $m_{b}=\frac{|a|^{2}}{M_{\mathrm{sol}}}$. This can be thought of as the minimal (two right-handed neutrino) predictive seesaw model since only four real parameters $m_{a}, m_{b}, n, \eta$ describe the entire neutrino sector (three neutrino masses as well as the PMNS matrix, in the diagonal charged lepton mass basis). As we shall see in the next section, $\eta$ is identified with the leptogenesis phase, while $m_{b}$ is identified with the neutrinoless double beta decay parameter $m_{e e}$.

\section{$7 \quad$ A numerical benchmark: $\operatorname{CSD}(3)$ with $\eta=2 \pi / 3$}

We now illustrate the success of the scheme by presenting numerical results for the neutrino mass matrix in eq. (6.5) for the particular choice of input parameters, namely $n=3$ and $\eta=2 \pi / 3,^{2}$

$$
m^{\nu}=m_{a}\left(\begin{array}{lll}
0 & 0 & 0 \\
0 & 1 & 1 \\
0 & 1 & 1
\end{array}\right)+m_{b} e^{i 2 \pi / 3}\left(\begin{array}{lll}
1 & 3 & 1 \\
3 & 9 & 3 \\
1 & 3 & 1
\end{array}\right) .
$$

This numerical benchmark was first presented in [25, 26]. In section 14 we will propose a simple LS model which provides a theoretical justification for this choice of parameters.

In table 1 we compare the above numerical benchmark resulting from the neutrino mass matrix in eq. (7.1) to the global best fit values from [38] (setting $m_{1}=0$ ). The 


\begin{tabular}{|c|c|c|c|c|c|c|c|c|}
\hline $\begin{array}{c}m_{a} \\
(\mathrm{meV})\end{array}$ & $\begin{array}{cc}m_{b} & \eta \\
(\mathrm{meV}) & (\mathrm{rad})\end{array}$ & $\begin{array}{c}\theta_{12} \\
\left(^{\circ}\right)\end{array}$ & $\begin{array}{l}\theta_{13} \\
\left({ }^{\circ}\right)\end{array}$ & $\begin{array}{c}\theta_{23} \\
\left(^{\circ}\right)\end{array}$ & $\begin{array}{c}\delta_{\mathrm{CP}} \\
\left(^{\circ}\right)\end{array}$ & $\begin{array}{c}m_{1} \\
(\mathrm{meV})\end{array}$ & $\begin{array}{c}m_{2} \\
(\mathrm{meV})\end{array}$ & $\begin{array}{c}m_{3} \\
(\mathrm{meV})\end{array}$ \\
\hline 26.57 & $2.684 \frac{2 \pi}{3}$ & 34.3 & 8.67 & 45.8 & -86.7 & 0 & 8.59 & 49.8 \\
\hline Value & from $[38]$ & $33.48_{-0.75}^{+0.78}$ & $8.50_{-0.21}^{+0.20}$ & $42.3_{-1.6}^{+3.0}$ & $-54_{-70}^{+39}$ & 0 & $8.66 \pm 0.10$ & $49.57 \pm 0.47$ \\
\hline
\end{tabular}

Table 1. Parameters and predictions for $\operatorname{CSD}(3)$ with a fixed phase $\eta=2 \pi / 3$ from [25]. In addition we predict $\beta=71.9^{\circ}$ which is not shown in the table since the neutrinoless double beta decay parameter is $m_{e e}=m_{b}=2.684 \mathrm{meV}$ for the above parameter set which is practically impossible to measure in the forseeable future. These predictions may be compared to the global best fit values from [38] (for $\left.m_{1}=0\right)$, given on the last line.

agreement between $\operatorname{CSD}(3)$ and data is within about one sigma for all the parameters, with similar agreement for the other global fits [39, 40].

Using the results in table 1, the baryon asymmetry of the Universe (BAU) resulting from $N_{1}=N_{\text {atm }}$ leptogenesis was estimated for this model [26]:

$$
Y_{B} \approx 2.5 \times 10^{-11} \sin \eta\left[\frac{M_{1}}{10^{10} \mathrm{GeV}}\right] .
$$

Using $\eta=2 \pi / 3$ and the observed value of $Y_{B}$ fixes the lightest right-handed neutrino mass:

$$
M_{1}=M_{\mathrm{atm}} \approx 3.9 \times 10^{10} \mathrm{GeV} .
$$

The phase $\eta$ determines the BAU via leptogenesis in eq. (7.2). In fact it controls the entire PMNS matrix, including all the lepton mixing angles as well as all low energy $\mathcal{C P}$ violation. The leptogenesis phase $\eta$ is therefore the source of all $\mathcal{C P}$ violation arising from this model, including $\mathcal{C P}$ violation in neutrino oscillations and in leptogenesis. There is a direct link between measurable and cosmological $\mathcal{C P}$ violation in this model and a correlation between the sign of the BAU and the sign of low energy leptonic $\mathcal{C P}$ violation. The leptogenesis phase is fixed to be $\eta=2 \pi / 3$ which leads to the observed excess of matter over antimatter for $M_{1} \approx 4.10^{10} \mathrm{GeV}$, yielding an observable neutrino oscillation phase $\delta_{\mathrm{CP}} \approx-\pi / 2$.

\section{Exact analytic results for lepton mixing angles}

We would like to understand the numerical success of the neutrino mass matrix analytically. In the following sections we shall derive exact analytic results for neutrino masses and PMNS parameters, for real continuous $n$, corresponding to the physical (light effective left-handed Majorana) neutrino mass matrix, in the diagonal charged lepton mass basis in eq. (6.5), which we reproduce below,

$$
m^{\nu}=m_{a}\left(\begin{array}{lll}
0 & 0 & 0 \\
0 & 1 & 1 \\
0 & 1 & 1
\end{array}\right)+m_{b} e^{i \eta}\left(\begin{array}{ccc}
1 & n & n-2 \\
n & n^{2} & n(n-2) \\
1 & n(n-2) & (n-2)^{2}
\end{array}\right) .
$$

\footnotetext{
${ }^{2}$ Note that the seesaw mechanism results in a light effective Majorana mass matrix given by the Lagrangian $\mathcal{L}_{\text {eff }}=-\frac{1}{2} \overline{\nu_{L}} m^{\nu} \nu_{L}^{c}+H . c$. . This corresponds to the convention of appendix A.
} 
Since this yields TM1 mixing as discussed above, it can be block diagonalised by the TB mixing matrix,

$$
m_{\text {block }}^{\nu}=U_{\mathrm{TB}}^{T} m^{\nu} U_{\mathrm{TB}}=\left(\begin{array}{ccc}
0 & 0 & 0 \\
0 & x & y \\
0 & y & z
\end{array}\right)
$$

where we find,

$$
x=3 m_{b} e^{i \eta}, \quad y=\sqrt{6} m_{b} e^{i \eta}(n-1), \quad z=|z| e^{i \phi_{z}}=2\left[m_{a}+m_{b} e^{i \eta}(n-1)^{2}\right]
$$

It only remains to put $m_{\text {block }}^{\nu}$ into diagonal form, with real positive masses, which can be done exactly analytically of course, since this is just effectively a two by two complex symmetric matrix,

$$
U_{\text {block }}^{T} m_{\text {block }}^{\nu} U_{\text {block }}=P_{3 \nu}^{*} R_{23 \nu}^{T} P_{2 \nu}^{*} m_{\text {block }}^{\nu} P_{2 \nu}^{*} R_{23 \nu} P_{3 \nu}^{*}=m_{\text {diag }}^{\nu}=\operatorname{diag}\left(0, m_{2}, m_{3}\right),
$$

where

$$
\begin{aligned}
P_{2 \nu} & =\left(\begin{array}{ccc}
1 & 0 & 0 \\
0 & e^{i \phi_{2}^{\nu}} & 0 \\
0 & 0 & e^{i \phi_{3}^{\nu}}
\end{array}\right) \\
P_{3 \nu} & =\left(\begin{array}{ccc}
e^{i \omega_{1}^{\nu}} & 0 & 0 \\
0 & e^{i \omega_{2}^{\nu}} & 0 \\
0 & 0 & e^{i \omega_{3}^{\nu}}
\end{array}\right)
\end{aligned}
$$

and

$$
R_{23 \nu}=\left(\begin{array}{ccc}
1 & 0 & 0 \\
0 & \cos \theta_{23}^{\nu} & \sin \theta_{23}^{\nu} \\
0 & -\sin \theta_{23}^{\nu} & \cos \theta_{23}^{\nu}
\end{array}\right) \equiv\left(\begin{array}{ccc}
1 & 0 & 0 \\
0 & c_{23}^{\nu} & s_{23}^{\nu} \\
0 & -s_{23}^{\nu} & c_{23}^{\nu}
\end{array}\right)
$$

where the angle $\theta_{23}^{\nu}$ is given exactly by,

$$
t \equiv \tan 2 \theta_{23}^{\nu}=\frac{2|y|}{|z| \cos (A-B)-|x| \cos B}
$$

where

$$
\tan B=\tan \left(\phi_{3}^{\nu}-\phi_{2}^{\nu}\right)=\frac{|z| \sin A}{|x|+|z| \cos A}
$$

where $x, y, z$ were defined in terms of input parameters in eq. (8.3) and

$$
A=\phi_{z}-\eta=\arg \left[m_{a}+m_{b} e^{i \eta}(n-1)^{2}\right]-\eta .
$$

Recall from eq. (A.2),

$$
V_{\nu_{L}} m^{\nu} V_{\nu_{L}}^{T}=m_{\text {diag }}^{\nu}=\operatorname{diag}\left(m_{1}, m_{2}, m_{3}\right)=\operatorname{diag}\left(0, m_{2}, m_{3}\right) .
$$

From eqs. (8.2), (8.4), (8.11) we identify,

$$
V_{\nu_{L}}=U_{\text {block }}^{T} U_{\mathrm{TB}}^{T}=P_{3 \nu}^{*} R_{23 \nu}^{T} P_{2 \nu}^{*} U_{\mathrm{TB}}^{T}, \quad V_{\nu_{L}}^{\dagger}=U_{\mathrm{TB}} U_{\text {block }}^{*}=U_{\mathrm{TB}} P_{2 \nu} R_{23 \nu} P_{3 \nu} .
$$


Explicitly we find from eq. (8.12), using eqs. (3.1), (8.5), (8.6), (8.7),

$$
V_{\nu_{L}}^{\dagger}=\left(\begin{array}{ccc}
\sqrt{\frac{2}{3}} & \frac{e^{i \phi_{2}^{\nu}}}{\sqrt{3}} c_{23}^{\nu} & \frac{e^{i \phi_{2}^{\nu}}}{\sqrt{3}} s_{23}^{\nu} \\
-\frac{1}{\sqrt{6}} & \frac{e^{i \phi_{2}^{\nu}}}{\sqrt{3}} c_{23}^{\nu}-\frac{e^{i \phi_{3}^{\nu}}}{\sqrt{2}} s_{23}^{\nu} & \frac{e^{i \phi_{3}^{\nu}}}{\sqrt{2}} c_{23}^{\nu}+\frac{e^{i \phi_{2}^{\nu}}}{\sqrt{3}} s_{23}^{\nu} \\
\frac{1}{\sqrt{6}} & -\frac{e^{i \phi_{2}^{\nu}}}{\sqrt{3}} c_{23}^{\nu}-\frac{e^{i \phi \phi_{3}^{\nu}}}{\sqrt{2}} s_{23}^{\nu} & \frac{e^{i \phi_{3}^{\nu}}}{\sqrt{2}} c_{23}^{\nu}-\frac{e^{i \phi_{2}^{\nu}}}{\sqrt{3}} s_{23}^{\nu}
\end{array}\right)\left(\begin{array}{ccc}
e^{i \omega_{1}^{\nu}} & 0 & 0 \\
0 & e^{i \omega_{2}^{\nu}} & 0 \\
0 & 0 & e^{i \omega_{3}^{\nu}}
\end{array}\right) .
$$

which takes the trimaximal form of eq. (3.3). Recall from eqs. (A.3), (A.4), (8.12), the PMNS matrix is given by,

$$
U=V_{E_{L}} V_{\nu_{L}}^{\dagger}
$$

Writing $\left(V_{\nu_{L}}^{\dagger}\right)_{i j}=e^{i \rho_{i j}^{\nu}}\left|\left(V_{\nu_{L}}^{\dagger}\right)_{i j}\right|$, and introducing two charged lepton phases, $\phi_{\mu}$ and $\phi_{\tau}$,

$$
U=\left(\begin{array}{ccc}
\sqrt{\frac{2}{3}} & \frac{1}{\sqrt{3}} c_{23}^{\nu} & \frac{1}{\sqrt{3}} s_{23}^{\nu} \\
e^{i \phi_{\mu}} \frac{-1}{\sqrt{6}} & e^{i \phi_{\mu}}\left(\frac{1}{\sqrt{3}} c_{23}^{\nu}-\frac{e^{i B}}{\sqrt{2}} s_{23}^{\nu}\right) & e^{i\left(\phi_{\mu}+\rho_{23}^{\nu}\right)}\left|\frac{e^{i B}}{\sqrt{2}} c_{23}^{\nu}+\frac{1}{\sqrt{3}} s_{23}^{\nu}\right| \\
e^{i \phi_{\tau}} \frac{1}{\sqrt{6}} e^{i \phi_{\tau}}\left(-\frac{1}{\sqrt{3}} c_{23}^{\nu}-\frac{e^{i B}}{\sqrt{2}} s_{23}^{\nu}\right) & e^{i\left(\phi_{\tau}+\rho_{33}^{\nu}\right)}\left|\frac{e^{i B}}{\sqrt{2}} c_{23}^{\nu}-\frac{1}{\sqrt{3}} s_{23}^{\nu}\right|
\end{array}\right)\left(\begin{array}{ccc}
e^{i \omega_{1}^{\nu}} & 0 & 0 \\
0 & e^{i\left(\phi_{2}^{\nu}+\omega_{2}^{\nu}\right)} & 0 \\
0 & 0 & e^{i\left(\phi_{2}^{\nu}+\omega_{3}^{\nu}\right)}
\end{array}\right)
$$

which can be compared to the PDG parameterisation in eq. (A.5), (A.6), (A.7),

$$
U=\left(\begin{array}{ccc}
c_{12} c_{13} & s_{12} c_{13} & s_{13} e^{-i \delta} \\
-s_{12} c_{23}-c_{12} s_{13} s_{23} e^{i \delta} & c_{12} c_{23}-s_{12} s_{13} s_{23} e^{i \delta} & c_{13} s_{23} \\
s_{12} s_{23}-c_{12} s_{13} c_{23} e^{i \delta} & -c_{12} s_{23}-s_{12} s_{13} c_{23} e^{i \delta} & c_{13} c_{23}
\end{array}\right)\left(\begin{array}{ccc}
e^{i \frac{\beta_{1}}{2}} & 0 & 0 \\
0 & e^{i \frac{\beta_{2}}{2}} & 0 \\
0 & 0 & 1
\end{array}\right)
$$

from which comparison we identify the physical PMNS lepton mixing angles by the exact expressions

$$
\begin{aligned}
\sin \theta_{13} & =\frac{1}{\sqrt{3}} s_{23}^{\nu}=\frac{1}{\sqrt{6}}\left(1-\sqrt{\frac{1}{1+t^{2}}}\right)^{1 / 2} \\
\tan \theta_{12} & =\frac{1}{\sqrt{2}} c_{23}^{\nu}=\frac{1}{\sqrt{2}}\left(1-3 \sin ^{2} \theta_{13}\right)^{1 / 2} \\
\tan \theta_{23} & =\frac{\left|\frac{e^{i B}}{\sqrt{2}} c_{23}^{\nu}+\frac{1}{\sqrt{3}} s_{23}^{\nu}\right|}{\left|\frac{e^{i B}}{\sqrt{2}} c_{23}^{\nu}-\frac{1}{\sqrt{3}} s_{23}^{\nu}\right|}=\frac{\left|1+\epsilon_{23}^{\nu}\right|}{\left|1-\epsilon_{23}^{\nu}\right|}
\end{aligned}
$$

where we have selected the negative sign for the square root in parentheses, applicable for the physical range of parameters, and defined

$$
\epsilon_{23}^{\nu} \equiv \sqrt{\frac{2}{3}} \tan \theta_{23}^{\nu} e^{-i B}=\sqrt{\frac{2}{3}} t^{-1}\left[\sqrt{1+t^{2}}-1\right] e^{-i B}
$$

expressing the results in terms of $t \equiv \tan 2 \theta_{23}^{\nu}$ and $B=\phi_{3}^{\nu}-\phi_{2}^{\nu}$ which were given in terms of input parameters in eqs. (8.8), (8.9), (8.10). The solar angle $\tan \theta_{12}$ approximately takes the TB value of $1 / \sqrt{2}$, to first order in $\sin \theta_{13}$. The atmospheric angle $\tan \theta_{23}$ is maximal when $B= \pm \pi / 2$ since then $\left|1+\epsilon_{23}^{\nu}\right|$ is equal to $\left|1-\epsilon_{23}^{\nu}\right|$. 


\section{$9 \quad$ Exact analytic results for neutrino masses}

The neutrino masses can be calculated from the block diagonal form of the neutrino mass matrix in eq. (8.2) which is diagonalised with real postive mass eigenvalues $m_{2}, m_{3}$ as in eq. (8.4). After forming the Hermitian combination (in terms of $x, y, z$ in eq. (8.3)),

$$
H_{\text {block }}^{\nu}=m_{\text {block }}^{\nu} m_{\text {block }}^{\nu \dagger}=\left(\begin{array}{ccc}
0 & 0 & 0 \\
0 & |x|^{2}+|y|^{2} & |x||y|+|y| e^{i \eta} z^{*} \\
0|x||y|+|y| e^{-i \eta} z & |y|^{2}+|z|^{2}
\end{array}\right)
$$

we diagonalise it by,

$$
U_{\text {block }}^{T} H_{\text {block }}^{\nu} U_{\text {block }}^{*}=\operatorname{diag}\left(0, m_{2}^{2}, m_{3}^{2}\right) .
$$

Then, by taking the Trace (T) and Determinant (D) of eq. (9.2), using eq. (9.1), we find

$$
\begin{aligned}
m_{2}^{2}+m_{3}^{2} & =T \equiv|x|^{2}+2|y|^{2}+|z|^{2} \\
m_{2}^{2} m_{3}^{2} & =D \equiv|x|^{2}|z|^{2}+|y|^{4}-2|x||y|^{2}|z| \cos A
\end{aligned}
$$

from which we extract the exact results for the neutrino masses,

$$
\begin{aligned}
& m_{3}^{2}=\frac{1}{2} T+\frac{1}{2} \sqrt{T^{2}-4 D} \\
& m_{2}^{2}=D / m_{3}^{2} \\
& m_{1}^{2}=0
\end{aligned}
$$

where we have selected the positive sign for the square root which is applicable for $m_{3}^{2}>m_{2}^{2}$. Furthermore, since $m_{3}^{2} \gg m_{2}^{2}$ (recall $m_{3}^{2} / m_{2}^{2} \approx 30$ ) we may approximate,

$$
\begin{aligned}
& m_{3}^{2} \approx T=|x|^{2}+2|y|^{2}+|z|^{2} \\
& m_{2}^{2} \approx D / T=\frac{|x|^{2}|z|^{2}+|y|^{4}-2|x||y|^{2}|z| \cos A}{|x|^{2}+2|y|^{2}+|z|^{2}}
\end{aligned}
$$

The sequential dominance (SD) approximation that the atmospheric right-handed neutrino dominates over the solar right-handed neutrino contribution to the seesaw mass matrix implies that $m_{a} \gg m_{b}$ and $|z| \gg|x|,|y|$ leading to

$$
\begin{aligned}
& m_{3}^{2} \approx|z|^{2} \quad \longrightarrow \quad m_{3} \approx 2 m_{a} \\
& m_{2}^{2} \approx|x|^{2} \quad \longrightarrow \quad m_{2} \approx 3 m_{b}
\end{aligned}
$$

using the definitions in eq. (8.3). The results in eqs. (9.10) and (9.11) are certainly very simple, but how accurate are they? For the $\operatorname{CSD}(3)$ numerical benchmark $m_{a} \approx 27 \mathrm{meV}$ and $m_{b} \approx 2.7 \mathrm{meV}$ gives $m_{3} \approx 50 \mathrm{meV}, m_{2} \approx 8.6 \mathrm{meV}$. We find $|z| \approx 46 \mathrm{meV},|x| \approx 8.0$ $\mathrm{meV}$ (and $|y| \approx 13 \mathrm{meV}$ ) which is a reasonable approximation. The SD approximations in eqs. (9.10) and (9.11) give, $m_{3} \approx 2 m_{a} \approx 54 \mathrm{meV}$ and $m_{2} \approx 3 m_{b} \approx 8.1 \mathrm{meV}$, accurate to say $10 \%$. The approximate results in eqs. (9.8), (9.9) are more accurate to say $3 \%$, with the results in eqs. (9.5), (9.6) being of course exact. 
The SD approximation in eqs. (9.10), (9.11) is both insightful and useful, since two of the three input parameters, namely $m_{a}$ and $m_{b}$, are immediately fixed by the two physical neutrino masses $m_{3}$ and $m_{2}$, which, for $m_{1}=0$, are identified as the square roots of the measured mass squared differences $\Delta m_{31}^{2}$ and $\Delta m_{21}^{2}$. This leaves, in the SD approximation, the only remaining parameters to be $n$ and the phase $\eta$, which, together, determine the entire PMNS mixing matrix ( 3 angles, and 2 phases). For example if $n=3$ and $\eta=2 \pi / 3$ were determined by some model, then the PMNS matrix would be determined uniquely, without any freedom, in the SD approximation. When searching for a best fit solution, the SD approximation in in eqs. (9.10), (9.11) is useful as a first approximation which enables the parameters $m_{a}$ and $m_{b}$ to be approximately determined by $\Delta m_{31}^{2}$ and $\Delta m_{21}^{2}$ since this may then be used as a starting point around which a numerical minimisation package can be run using the exact results for the neutrino masses in eqs. (9.5), (9.6) together with the exact results for the lepton mixing angles in eqs. (8.17), (8.18), (8.19).

\section{Exact analytic results for $\mathrm{CP}$ violation}

In this model the Majorana phase $\beta_{1}$ is unphysical since $m_{1}=0$ so the only physical Majorana phase is $\beta \equiv \beta_{2}$. By comparing eqs. (8.15), (8.16) the physical PMNS phases are then identified as

$$
\begin{aligned}
\delta & =\rho_{23}^{\nu}+\phi_{\mu}=\rho_{33}^{\nu}+\phi_{\tau} \\
\frac{\beta}{2} & =\omega_{2}^{\nu}-\omega_{3}^{\nu}-\delta
\end{aligned}
$$

Extracting the value of the physical phases $\delta$ and $\beta$ in terms of input parameters is rather cumbersome and it is better to use the Jarlskog and Majorana invariants in order to do this.

\subsection{The Jarlskog invariant}

The Jarlskog invariant $J$ [41] can be derived starting from the invariant [42, 43],

$$
I_{1}=\operatorname{Tr}\left[H^{\nu}, H^{E}\right]^{3}=\operatorname{Tr}\left(\left[H^{\nu} H^{E}-H^{E} H^{\nu}\right]^{3}\right)
$$

where the Hermitian matrices are defined as

$$
H^{\nu}=m^{\nu} m^{\nu \dagger}, \quad H^{E}=m^{E} m^{E \dagger}
$$

In our conventions of section A, by explicit calculation one can verify the well known result that

$$
I_{1}=-6 i \Delta m_{\nu}^{6} \Delta m_{E}^{6} J
$$

where

$$
\begin{aligned}
\Delta m_{\nu}^{6} & =\left(m_{3}^{2}-m_{1}^{2}\right)\left(m_{2}^{2}-m_{1}^{2}\right)\left(m_{3}^{2}-m_{2}^{2}\right)=m_{3}^{2} m_{2}^{2} \Delta m_{32}^{2} \\
\Delta m_{E}^{6} & =\left(m_{\tau}^{2}-m_{e}^{2}\right)\left(m_{\mu}^{2}-m_{e}^{2}\right)\left(m_{\tau}^{2}-m_{\mu}^{2}\right) \\
J & =\Im\left[U_{11} U_{22} U_{12}^{*} U_{21}^{*}\right]=s_{12} c_{12} s_{13} c_{13}^{2} s_{23} c_{23} \sin \delta
\end{aligned}
$$


The above results show that $I_{1}$ is basis invariant since it can be expressed in terms of physical masses and PMNS parameters. We are therefore free to evaluate $I_{1}$ in any basis. For example, in the diagonal charged lepton mass basis, one can shown that the quantity in eq. (10.3) becomes,

$$
I_{1}=\operatorname{Tr}\left[H^{\nu}, H^{E}\right]^{3}=6 i \Delta m_{E}^{6} \Im\left(H_{12}^{\nu *} H_{13}^{\nu} H_{23}^{\nu *}\right)
$$

where in eq. (10.9), the Hermitian matrix $H^{\nu}=m^{\nu} m^{\nu \dagger}$ involves the neutrino mass matrix $m^{\nu}$ in the basis where the charged lepton mass matrix is diagonal, i.e. the basis of eq. (6.5), where we find

$$
\Im\left(H_{12}^{\nu *} H_{13}^{\nu} H_{23}^{\nu *}\right)=24 m_{a}^{3} m_{b}^{3}(n-1) \sin \eta
$$

From eqs. (10.5) and (10.9) we find, after equating these two expressions,

$$
J=-\frac{\Im\left(H_{12}^{\nu *} H_{13}^{\nu} H_{23}^{\nu *}\right)}{\Delta m_{\nu}^{6}}=-\frac{24 m_{a}^{3} m_{b}^{3}(n-1) \sin \eta}{m_{3}^{2} m_{2}^{2} \Delta m_{32}^{2}}
$$

where we have used eqs. (10.6) and (10.10). From eqs. (10.8) and (10.11) we find, after equating these two expressions, we find the exact relation

$$
\sin \delta=-\frac{24 m_{a}^{3} m_{b}^{3}(n-1) \sin \eta}{m_{3}^{2} m_{2}^{2} \Delta m_{32}^{2} s_{12} c_{12} s_{13} c_{13}^{2} s_{23} c_{23}}
$$

Note the minus sign in eq. (10.12), which means that, for $n>1$, the $\operatorname{sign}$ of $\sin \delta$ takes the opposite value to the sign of $\sin \eta$, in the convention we use to write our neutrino mass matrix in section A. Since the denominator of eq. (10.12) may be expressed in terms of input parameters, using the exact results for the neutrino masses in eqs. (9.5), (9.6) together with the exact results for the lepton mixing angles in eqs. (8.17), (8.18), (8.19), it is clear that eq. (10.12) gives $\sin \delta$ in terms of input parameters in both the numerator and demominator.

In the SD approximation in eqs. (9.10), (9.11) we find from eq. (10.11),

$$
J \approx-\frac{1}{9} \frac{m_{2}}{m_{3}}(n-1) \sin \eta
$$

where the minus sign in eq. (10.13) again clearly shows the anti-sign correlation of $\sin \delta$ and $\sin \eta$, where $\eta$ is the input phase which appears in the neutrino mass matrix in eq. (6.5) and leptogenesis in eq. (7.2). In other words the BAU is proportional to $-\sin \delta$ if the lightest right-handed neutrino is the one dominantly responsible for the atmospheric neutrino mass $N_{1}=N_{\text {atm }}$. In this case the observed matter Universe requires $\sin \delta$ to be negative in order to generate a positive BAU. It is interesting to note that, up to a negative factor, the sine of the leptogenesis phase $\eta$ is equal to the sine of the oscillation phase $\delta$, so the observation the $\mathrm{CP}$ violation in neutrino oscillations is directly responsible for the $\mathrm{CP}$ violation in the early Universe, in the LS model. 


\subsection{The Majorana invariant}

The Majorana invariant may be defined by [44],

$$
I_{2}=\Im \operatorname{Tr}\left[H^{E} m^{\nu} m^{\nu \dagger} m^{\nu} H^{E *} m^{\nu \dagger}\right]
$$

In our conventions of section A, this may be written,

$$
I_{2}=\Im \operatorname{Tr}\left[U^{\dagger} H_{\text {diag }}^{E} U m_{\text {diag }}^{\nu 3} U^{T} H_{\text {diag }}^{E} U^{*} m_{\text {diag }}^{\nu}\right]
$$

By explicit calculation we find an exact but rather long expression which is basis invariant since it involves physical masses and PMNS parameters. We do not show the result here since it is rather long and not very illuminating and also not so relevant since Majorana $\mathrm{CP}$ violation is not going to be measured for a very long time. However, since $m_{e}^{2} \ll m_{\mu}^{2} \ll$ $m_{\tau}^{2}$, we may neglect $m_{e}^{2}$ and $m_{\mu}^{2}$ compared to $m_{\tau}^{2}$, and also drop $s_{13}^{2}$ terms, to give the compact result,

$$
I_{2} \approx-m_{\tau}^{4} m_{2} m_{3} \Delta m_{32}^{2} c_{13}^{2} c_{23}^{2}\left[c_{12}^{2} s_{23}^{2}(\sin \beta)+2 c_{12} c_{23} s_{12} s_{13} s_{23} \sin (\beta+\delta)\right] .
$$

Notice that $I_{2}$ is zero if both $\beta$ and $\delta$ are zero or $\pi$, so it is indeed sensitive to Majorana $\mathrm{CP}$ violation arising from $\beta$. Indeed $I_{2}$ is roughly proportional to $\sin \beta$ if the $s_{13}$ term is also neglected,

$$
I_{2} \approx-m_{\tau}^{4} m_{2} m_{3} \Delta m_{32}^{2} c_{13}^{2} c_{23}^{2} c_{12}^{2} s_{23}^{2}(\sin \beta) .
$$

We are free to evaluate $I_{2}$ in any basis. For example, in the diagonal charged lepton mass basis, the quantity in eq. (10.14) becomes,

$$
I_{2}=\Im \operatorname{Tr}\left[H_{\mathrm{diag}}^{E} m^{\nu} m^{\nu \dagger} m^{\nu} H_{\mathrm{diag}}^{E} m^{\nu \dagger}\right]
$$

where in eq. (10.18), the neutrino mass matrix $m^{\nu}$ is in the basis where the charged lepton mass matrix is diagonal, i.e. the basis of eq. (6.5). Evaluating eq. (10.18) we find the exact result,

$$
I_{2}=m_{a} m_{b} \sin \eta\left[-4 m_{a}^{2}\left(m_{\tau}^{2}-m_{\mu}^{2}\right)^{2}+m_{b}^{2}\left(m_{\tau}^{2}(2 n+1)(n-2)-m_{\mu}^{2} n(2 n-5)-2 m_{e}^{2}(n-1)\right)^{2}\right]
$$

If we neglect $m_{e}^{2}$ and $m_{\mu}^{2}$, eq. (10.19) becomes approximately,

$$
I_{2} \approx m_{\tau}^{4} m_{a} m_{b} \sin \eta\left[-4 m_{a}^{2}+m_{b}^{2}(2 n+1)^{2}(n-2)^{2}\right] .
$$

From eqs. (10.17) and (10.20) we find, after equating these two expressions, one finds an approximate formula for the sine of the Majorana phase,

$$
\sin \beta \approx \frac{m_{a} m_{b}\left[4 m_{a}^{2}-m_{b}^{2}(2 n+1)^{2}(n-2)^{2}\right] \sin \eta}{m_{2} m_{3} \Delta m_{32}^{2} c_{13}^{2} c_{23}^{2} c_{12}^{2} s_{23}^{2}}
$$

Since the denominator of eq. (10.21) may be expressed in terms of input parameters, using the exact results for the neutrino masses in eqs. (9.5), (9.6) together with the exact results for the lepton mixing angles in eqs. (8.17), (8.18), (8.19), it is clear that eq. (10.21) gives 
$\sin \beta$ in terms of input parameters in both the numerator and demominator. For low values of $n$ (e.g. $n=3$ ) the sign of $\sin \beta$ is the same as the $\operatorname{sign}$ of $\sin \eta$ and hence the opposite of the sign of $\sin \delta$ given by eq. (10.12).

It is worth recalling at this point that our Majorana phases are in the convention of eq. (A.7), namely $P=\operatorname{diag}\left(e^{i \frac{\beta_{1}}{2}}, e^{i \frac{\beta_{2}}{2}}, 1\right)$, where we defined $\beta=\beta_{2}$ and $\beta_{1}$ is unphysical since $m_{1}=0$. In another common convention the Majorana phases are by given by $P=\operatorname{diag}\left(1, e^{i \frac{\alpha_{21}}{2}}, e^{i \frac{\alpha_{31}}{2}}\right)$, which are related to ours by $\alpha_{21}=\beta_{2}-\beta_{1}$ and $\alpha_{31}=-\beta_{1}$. For the case at hand, where $m_{1}=0$, one finds $\beta=\alpha_{21}-\alpha_{31}$ to be the only Majorana phase having any physical significance (e.g. which enters the formula for neutrinoless double beta decay). This is the phase given by eq. (10.21).

Eq. (10.21) is independent of $s_{13}$ since we have dropped those terms. It is only therefore expected to be accurate to about $15 \%$, which is acceptable, given that the Majorana phase $\beta$ is practically impossible to measure in the forseeable future for the case of a normal mass hierarchy with the lightest neutrino mass $m_{1}=0$. However, if it becomes necessary in the future to have a more accurate result, this can be obtained by equating eq. (10.16) with eq. (10.20), which would yield an implicit formula for $\beta$ which is accurate to about $3 \%$ :

$$
\sin \beta+2 c_{12}^{-1} c_{23} s_{12} s_{13} s_{23}^{-1} \sin (\beta+\delta) \approx \frac{m_{a} m_{b}\left[4 m_{a}^{2}-m_{b}^{2}(2 n+1)^{2}(n-2)^{2}\right] \sin \eta}{m_{2} m_{3} \Delta m_{32}^{2} c_{13}^{2} c_{23}^{2} c_{12}^{2} s_{23}^{2}} .
$$

\section{Exact sum rules}

The formulas in the previous section give the observable physical neutrino masses and the PMNS angles and phases in terms of fewer input parameters $m_{a}, m_{b}, n$ and $\eta$. In particular, the exact results for the neutrino masses are given in eqs. (9.5), (9.6), (9.7), the exact results for the lepton mixing angles are given in eqs. (8.17), (8.18), (8.19) and the exact result for the CP violating Dirac oscillation phase is given in eq. (10.12), while the Majorana phase is given approximately by eq. (10.21). These 8 equations for the 8 observables cannot be inverted to give the 4 input parameters in terms of the 8 physical parameters since there are clearly fewer input parameters than observables. On the one hand, this is good, since it means that the littlest seesaw has 4 predictions, on the other hand it does mean that we have to deal with a 4 dimensional input parameter space. Later we shall impose additional theoretical considerations, which shall reduce this parameter space to just 2 input parameters, yielding 6 predictions, but for now we consider the 4 input paramaters. In any case it is not obvious that one can derive any sum rules where input parameters are eliminated, and only relations between physical observables remain.

Nevertheless in this model there are such sum rules, i.e. relations between physical observables not involving the input parameters. An example of such a sum rule is eq. (8.18), which we give below in three equivalent exact forms,

$$
\tan \theta_{12}=\frac{1}{\sqrt{2}} \sqrt{1-3 s_{13}^{2}} \quad \text { or } \quad \sin \theta_{12}=\frac{1}{\sqrt{3}} \frac{\sqrt{1-3 s_{13}^{2}}}{c_{13}} \quad \text { or } \quad \cos \theta_{12}=\sqrt{\frac{2}{3}} \frac{1}{c_{13}}
$$

This sum rule is in fact common to all TM1 models, and is therefore also applicable to the LS models which predict TM1 mixing. Similarly TM1 predicts the so called atmospheric 
sum rule, also applicable to the LS models. This arises from the fact that the first column of the PMNS matrix is the same as the first column of the TB matrix in eq. (3.1). Indeed, by comparing the magnitudes of the elements in the first column of eq. (8.15) to those in the first column of eq. (8.16), we obtain,

$$
\begin{aligned}
& \left|U_{e 1}\right|=c_{12} c_{13}=\sqrt{\frac{2}{3}} \\
& \left|U_{\mu 1}\right|=\left|-s_{12} c_{23}-c_{12} s_{13} s_{23} e^{i \delta}\right|=\sqrt{\frac{1}{6}} \\
& \left|U_{\tau 1}\right|=\left|s_{12} s_{23}-c_{12} s_{13} c_{23} e^{i \delta}\right|=\sqrt{\frac{1}{6}}
\end{aligned}
$$

Eq. (11.2) is equivalent to eq. (11.1) while eqs. (11.3) and (11.4) lead to equivalent mixing sum rules which can be expressed as an exact relation for $\cos \delta$ in terms of the other lepton mixing angles [31, 32],

$$
\cos \delta=-\frac{\cot 2 \theta_{23}\left(1-5 s_{13}^{2}\right)}{2 \sqrt{2} s_{13} \sqrt{1-3 s_{13}^{2}}}
$$

Note that, for maximal atmospheric mixing, $\theta_{23}=\pi / 4$, we see that $\cot 2 \theta_{23}=0$ and therefore this sum rule predicts $\cos \delta=0$, corresponding to maximal $\mathrm{CP}$ violation $\delta=$ $\pm \pi / 2$. The prospects for testing the TM1 atmospheric sum rules eqs. (11.1), (11.5) in future neutrino facilities was discussed in $[45,46]$.

The LS model also the predicts additional sum rules beyond the TM1 sum rules that arise from the structure of the Dirac mass matrix in eq. (6.2). Recalling that the PMNS matrix is written in eq. (A.5) as $U=V P$, where $V$ is the the CKM-like part and $P$ contains the Majorana phase $\beta$, the LS sum rules are [20],

$$
\begin{aligned}
\frac{\left|V_{\mu 3} V_{e 2}-V_{\mu 2} V_{e 3}\right|}{\left|V_{\tau 3} V_{e 2}-V_{\tau 2} V_{e 3}\right|} & =1 \\
\frac{\left|e^{i \beta} m_{2} V_{\mu 2} V_{e 2}+m_{3} V_{\mu 3} V_{e 3}\right|}{\left|e^{i \beta} m_{2} V_{e 2}^{2}+m_{3} V_{e 3}^{2}\right|} & =n \\
\frac{\left|e^{i \beta} m_{2} V_{\tau 2} V_{e 2}+m_{3} V_{\tau 3} V_{e 3}\right|}{\left|e^{i \beta} m_{2} V_{e 2}^{2}+m_{3} V_{e 3}^{2}\right|} & =n-2
\end{aligned}
$$

where the sum rule in eq. (11.6) is independent of both $n$ and $\beta$. We emphasise again that the matrix elements $V_{\alpha i}$ refer to the first matrix on the right-hand side of eq. (8.16) (i.e. without the Majorana matrix). Of course similar relations apply with $U$ replacing $V$ everywhere and the Majorana phase $\beta$ disappearing, being absorbed into the PMNS matrix $U$, but we prefer to exhibit the Majorana phase dependence explicitly. However the LS sum rule in eq. (11.6) is equivalent to the TM1 sum rule in eq. (11.5), as seen by explicit calculation. The other LS sum rules in eqs. (11.7) and (11.8) involve the phase $\beta$ and are not so interesting.

\section{The reactor and atmospheric angles}

Since the solar angle is expected to be very close to its tribimaximal value, according to the TM1 sum rules in eq. (11.1), independent of the input parameters, in this section we 
focus on the analytic predictions for the reactor and atmospheric angles, starting with the accurately measured reactor angle which is very important for pinning down the input parameters of the LS model.

\subsection{The reactor angle}

The exact expression for the reactor angle in eq. (8.17) is summarised below,

$$
\sin \theta_{13}=\frac{1}{\sqrt{6}}\left(1-\sqrt{\frac{1}{1+t^{2}}}\right)^{1 / 2},
$$

where from eqs. (8.3), (8.8), (8.9), (8.10),

$$
t=\frac{2 \sqrt{6} m_{b}(n-1)}{2\left|m_{a}+m_{b} e^{i \eta}(n-1)^{2}\right| \cos (A-B)-3 m_{b} \cos B}
$$

where

$$
\tan B=\frac{2\left|m_{a}+m_{b} e^{i \eta}(n-1)^{2}\right| \sin A}{3 m_{b}+2\left|m_{a}+m_{b} e^{i \eta}(n-1)^{2}\right| \cos A}
$$

and

$$
A=\arg \left[m_{a}+m_{b} e^{i \eta}(n-1)^{2}\right]-\eta .
$$

The above results are exact and necessary for precise analysis of the model, especially for large $n$ (where $n$ is in general a real and continuous number). We now proceed to derive some approxinate formulae which can give useful insight.

The SD approximations in eqs. (9.10), (9.11) show that $m_{b} / m_{a} \approx(2 / 3) m_{2} / m_{3}$. This suggests that we can make an expansion in $m_{b} / m_{a}$, or simply drop $m_{b}$ compared to $m_{a}$, as a leading order approximation, which implies $\tan B \approx \tan A$ and hence $\cos (A-B) \approx 1$. Thus eq. (12.2) becomes,

$$
t \approx \sqrt{6} \frac{m_{b}(n-1)}{\left|m_{a}+m_{b} e^{i \eta}(n-1)^{2}\right|}
$$

where we have kept the term proportional to $m_{b}(n-1)^{2}$, since the smallness of $m_{b}$ may be compensated by the factor $(n-1)^{2}$ for $n>1$. Eq. 12.5 shows that $t \ll 1$, hence we may expand eq. (12.1) to leading order in $t$,

$$
\sin \theta_{13} \approx \frac{t}{2 \sqrt{3}}+\mathcal{O}\left(t^{3}\right)
$$

Hence combining eqs. (12.5) and (12.6), we arrive at our approximate form for the sine of the reactor angle,

$$
\sin \theta_{13} \approx \frac{1}{\sqrt{2}} \frac{m_{b}(n-1)}{\left|m_{a}+m_{b} e^{i \eta}(n-1)^{2}\right|} .
$$

For low values of $(n-1)$ such that $m_{b}(n-1)^{2} \ll m_{a}$, eq. (12.7) simplifies to,

$$
\sin \theta_{13} \approx(n-1) \frac{\sqrt{2}}{3} \frac{m_{2}}{m_{3}}
$$


using the SD approximations in eqs. (9.10), (9.11) that $m_{b} / m_{a} \approx(2 / 3) m_{2} / m_{3}$, valid to $10 \%$ accuracy. For example, the result shows that for the original CSD [18], where $n=1$, implies $\sin \theta_{13}=0$, while for $\operatorname{CSD}(2)$ [19] (i.e. $n=2$ ) we have $\sin \theta_{13} \approx \frac{\sqrt{2}}{3} \frac{m_{2}}{m_{3}}$, leading to $\theta_{13} \approx 4.7^{\circ}$ which is too small. For $\operatorname{CSD}(3)$ [20] (i.e. $n=3$ ) we have $\sin \theta_{13} \approx \frac{2 \sqrt{2}}{3} \frac{m_{2}}{m_{3}}$, leading to $\theta_{13} \approx 9.5^{\circ}$, in rough agreement with the observed value of $\theta_{13} \approx 8.5^{\circ}$, within the accuracy of our approximations. We conclude that these results show how $\sin \theta_{13} \sim \mathcal{O}\left(m_{2} / m_{3}\right)$ can be achieved, with values increasing with $n$, and confirm that $n \approx 3$ gives the best fit to the reactor angle. We emphasise that the approximate formula in eq. (12.8) has not been written down before, and that the exact results in eqs. (12.1), (12.2), (12.3), (12.4) are also new and in perfect agreement with the numerical results in table 1.

\subsection{The atmospheric angle}

The exact expression for the atmospheric angle in eq. (8.19) is summarised below,

$$
\tan \theta_{23}=\frac{\left|1+\epsilon_{23}^{\nu}\right|}{\left|1-\epsilon_{23}^{\nu}\right|}
$$

where

$$
\epsilon_{23}^{\nu}=\sqrt{\frac{2}{3}} t^{-1}\left[\sqrt{1+t^{2}}-1\right] e^{-i B}
$$

and $t$ and $B$ were summarised in eqs. (12.2), (12.3), (12.4). The above results, which are exact, show that the atmospheric angle is maximal for $B \approx \pm \pi / 2$, as noted previously.

We may expand eq. (12.10) to leading order in $t$,

$$
\epsilon_{23}^{\nu} \approx \frac{t}{\sqrt{6}} e^{-i B}+\mathcal{O}\left(t^{3}\right)
$$

Hence combining eqs. (12.9), (12.11), we arrive at an approximate form for the tangent of the atmospheric angle,

$$
\tan \theta_{23} \approx \frac{\left|1+\frac{t}{\sqrt{6}} e^{-i B}\right|}{\left|1-\frac{t}{\sqrt{6}} e^{-i B}\right|} \approx 1+2 \frac{t}{\sqrt{6}} \cos B,
$$

where $t$ was approximated in eq. (12.5). We observed earlier that, for $m_{b} \ll m_{a}, \tan B \approx$ $\tan A$ and hence $A \approx B$. Unfortunately it is not easy to obtain a reliable approximation for $A$ in eq. (12.4), unless $n \gtrsim 1$ in which case $A \approx-\eta$. However, for $n-1$ significantly larger than unity, this is not a good approximation. For example for $n=3$ from eq. (12.4) we have,

$$
A=\arg \left[m_{a}+4 m_{b} e^{i \eta}\right]-\eta
$$

Taking $m_{b} / m_{a}=1 / 10$, this gives

$$
A=\arg \left[1+0.4 e^{i \eta}\right]-\eta
$$

which shows that $A \approx-\eta$ is not a good approximation even though $m_{b} \ll m_{a}$. If we set, for example, $\eta=2 \pi / 3$, as in table 1 , then eq. (12.14) gives

$$
A=0.41-2 \pi / 3 \approx-0.53 \pi
$$


which happens to be close to $-\pi / 2$. Hence, since $A \approx B$, this choice of parameters implies $\cos B \approx 0$, leading to approximately maximal atmospheric mixing from eq. (12.12), as observed in table 1. At this point it is also worth recalling that for maximal atmospheric mixing, the TM1 sum rule in eq. (11.5) predicts that the cosine of the CP phase $\delta$ to be zero, corresponding to maximal Dirac CP violation $\delta= \pm \pi / 2$, as approximately found in table 1.

\section{CSD(3) vacuum alignments from $S_{4}$}

We saw from the discussion of the reactor angle, and in table 1, that the solar alignment in eq. (5.2) for the particular choice $n=3$ was favoured. In this section we show how the desired alignments for $n=3$ can emerge from $S_{4}$ due to residual symmetries. Although the charged lepton alignments we discuss were also obtained previously from $A_{4}$ [20], the neutrino alignments in eq. (5.2) for $n=3$ were not previously obtained from residual symmetries, and indeed we will see that they will arise from group elements which appear in $S_{4}$ but not $A_{4}$.

We first summarise the vacuum alignments that we desire:

$$
\left\langle\phi_{\mathrm{atm}}\right\rangle=v_{\mathrm{atm}}\left(\begin{array}{l}
0 \\
1 \\
1
\end{array}\right), \quad\left\langle\phi_{\mathrm{sol}}\right\rangle=v_{\mathrm{sol}}\left(\begin{array}{l}
1 \\
3 \\
1
\end{array}\right),
$$

in the neutrino sector as in eq. (5.2) with $n=3$, and,

$$
\left\langle\varphi_{e}\right\rangle=v_{e}\left(\begin{array}{l}
1 \\
0 \\
0
\end{array}\right), \quad\left\langle\varphi_{\mu}\right\rangle=v_{\mu}\left(\begin{array}{l}
0 \\
1 \\
0
\end{array}\right), \quad\left\langle\varphi_{\tau}\right\rangle=v_{\tau}\left(\begin{array}{l}
0 \\
0 \\
1
\end{array}\right) .
$$

in the charged lepton sector.

For comparison we also give the tribimaximal alignments in eq. (4.2):

$$
\left\langle\phi_{1}\right\rangle=v_{1}\left(\begin{array}{c}
2 \\
-1 \\
1
\end{array}\right), \quad\left\langle\phi_{2}\right\rangle=v_{2}\left(\begin{array}{c}
1 \\
1 \\
-1
\end{array}\right), \quad\left\langle\phi_{3}\right\rangle=v_{3}\left(\begin{array}{l}
0 \\
1 \\
1
\end{array}\right)
$$

We first observe that the charged lepton and the tribimaximal alignments individually preserve some remnant symmetry of $S_{4}$, whose triplet representations are displayed explicitly in appendix B. If we regard $\varphi_{e}, \varphi_{\mu}, \varphi_{\tau}$ as each being a triplet 3 of $S_{4}$, then they each correspond to a different symmetry conserving direction of $S_{4}$, with,

$$
a_{2}\left\langle\varphi_{e}\right\rangle=\left\langle\varphi_{e}\right\rangle, \quad a_{3}\left\langle\varphi_{\mu}\right\rangle=\left\langle\varphi_{\mu}\right\rangle, \quad a_{4}\left\langle\varphi_{\tau}\right\rangle=\left\langle\varphi_{\tau}\right\rangle
$$

One may question the use of different residual symmetry generators of $S_{4}$ to enforce the different charged lepton vacuum alignments. However, this is analagous to what is usually assumed in the direct model building approach when one says that the charged lepton sector preserves one residual symmetry, while the neutrino sector preserves another residual 
symmetry. In the direct case, it is clear that the lepton Lagrangian as a whole completely breaks the family symmetry, even though the charged lepton and neutrino sectors preserve different residual symmetries. In the indirect case here, we are taking this argument one step further, by saying that the electron, muon and tau sectors preserve different residual symmetries, while the charged lepton Lagrangian as a whole completely breaks the family symmetry. However the principle is the same as in the direct models, namely that different sectors of the Lagrangian preserve different residual subgroups of the family symmetry.

The tribimaximal alignment $\left\langle\phi_{2}\right\rangle$ is enforced by a combination of $d_{2}$ and $f_{1}$ being conserved,

$$
d_{2}\left\langle\phi_{2}\right\rangle=\left\langle\phi_{2}\right\rangle, \quad f_{1}\left\langle\phi_{2}\right\rangle=\left\langle\phi_{2}\right\rangle,
$$

which suggests that $\phi_{2}$ should be also identified as a triplet 3 of $S_{4}$. On the other hand, the tribimaximal alignment $\left\langle\phi_{3}\right\rangle$ (which is the same as the atmospheric alignment $\left\langle\phi_{\text {atm }}\right\rangle$ ) may be enforced by symmetry if $\phi_{3}$ (i.e. $\phi_{\text {atm }}$ ) is in the $\mathbf{3}^{\prime}$ representation, since then we see that,

$$
d_{2}^{\prime}\left\langle\phi_{3}\right\rangle=\left\langle\phi_{3}\right\rangle, \text { or } d_{2}^{\prime}\left\langle\phi_{\text {atm }}\right\rangle=\left\langle\phi_{\text {atm }}\right\rangle .
$$

As in the case of the charged lepton sector, we see that different parts of the neutrino sector will preserve different residual subgroups of the family symmetry $S_{4}$ for the tribimaximal alignments $\left\langle\phi_{2}\right\rangle$ and $\left\langle\phi_{3}\right\rangle=\left\langle\phi_{\text {atm }}\right\rangle$.

In order to obtain the alignments $\left\langle\phi_{1}\right\rangle$ and $\left\langle\phi_{\text {sol }}\right\rangle$ we must depart from the idea of residual symmetries and resort to dynamical terms in the potential that enforce orthogonality, as discussed in section 5. However, once the tribimaximal alignments $\left\langle\phi_{2}\right\rangle$ and $\left\langle\phi_{3}\right\rangle$ have been accomplished, the remaining tribimaximal alignment $\left\langle\phi_{1}\right\rangle$ is simple to obtain, see figure 1. Similarly the general solar alignment in eq. (5.2) then follows from the orthogonality to $\left\langle\phi_{1}\right\rangle$, as is also clear from figure 1 .

We now observe that the particular solar alignment $\left\langle\phi_{\text {sol }}\right\rangle$ in eq. (13.1) can be natually enforced by a symmetry argument if $\phi_{\text {sol }}$ is a triplet $\mathbf{3}$ of $S_{4}$ since then,

$$
f_{1}\left\langle\phi_{\text {sol }}\right\rangle=\left\langle\phi_{\text {sol }}\right\rangle,
$$

which by itself constrains the alignment to be $(1, m, 1)$, for continuous real $m$. However orthogonality to $\left\langle\phi_{1}\right\rangle$ further constrains the alignment to be $(1, n, n-2)$, for continuous real $n$. Taken together, the constrained forms $(1, n, n-2)$ and $(1, m, 1)$, fix $n=m$ and $n-2=1$, and hence $n=m=3$, corresponding to the alignment $(1,3,1)$ as desired in eq. (13.1).

To summarise we see that the desired alignments in eqs. (13.1) and 13.2 emerge naturally from the residual symmetries of $S_{4}$, together with the simple orthogonality conditions which can be readily obtained in models as in eq. (5.3). The residual $S_{4}$ symmetries involved in the various physical sectors of the model are summarised by the starfish shaped diagram in figure 2 .

\section{A benchmark model with $S_{4} \times Z_{3} \times Z_{3}^{\prime}$}

We now present a model based on $S_{4} \times Z_{3} \times Z_{3}^{\prime}$, which can reproduce the numerical benchmark discussed in section 7 . The $S_{4}$ will help produce the vacuum alignments with 


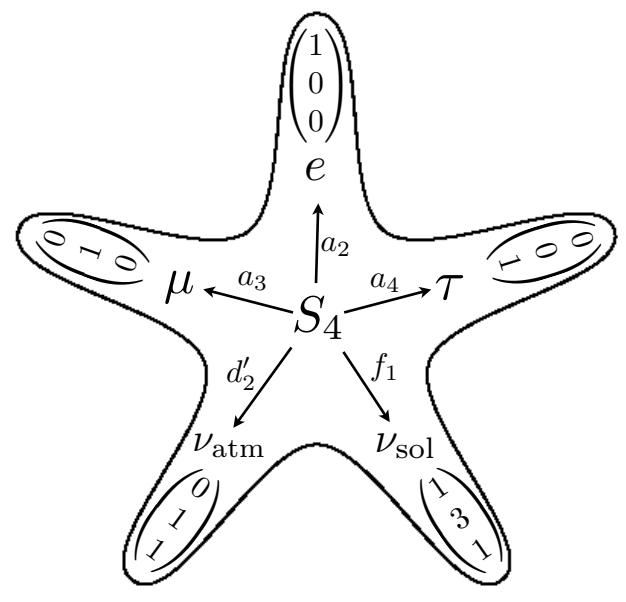

Figure 2. This starfish diagram summarises the residual $S_{4}$ symmetries respected by the vacuum alignments associated with the various physical sectors of the model. The residual symmetries are given by the preserved group elements $a_{i}, d_{2}^{\prime}, f_{1}$ defined in appendix B.

$n=3$, as discussed in the previous section, the $Z_{3}$ will help to fix $\eta=2 \pi / 3$ while the $Z_{3}^{\prime}$ will be responsible for the charged lepton mass hierarchy. This will yield the most predictive and successful version of the LS model, corresponding to the numerical results in table 1, perfectly reproduced by the exact analytic results, where the two remaining free parameters $m_{a}$ and $m_{b}$ are used to fix the neutrino mass squared differences. The entire PMNS matrix then emerges as a parameter free prediction, corresponding to the $\operatorname{CSD}(3)$ benchmark discussed in section 7 .

With the alignments in eqs. (13.1) and (13.2), arising as a consequence of $S_{4}$ residual symmetry, summarised by the starfish diagram in figure 2 , together with simple orthogonality conditions, as further discussed in the next section, we may write down the superpotential of the starfish lepton model, as a supersymmetric version ${ }^{3}$ of the LS Lagrangian in eq. (6.1),

$$
\begin{aligned}
W_{S_{4}}^{\mathrm{yuk}}= & \frac{1}{\Lambda} H_{2}\left(L \cdot \phi_{\mathrm{atm}}\right) \nu_{\mathrm{atm}}^{c}+\frac{1}{\Lambda} H_{2}\left(L . \phi_{\mathrm{sol}}\right) \nu_{\mathrm{sol}}^{c}+\xi_{\mathrm{atm}} \nu_{\mathrm{atm}}^{c} \nu_{\mathrm{atm}}^{c}+\xi_{\mathrm{sol}} \nu_{\mathrm{sol}}^{c} \nu_{\mathrm{sol}}^{c} \\
& +\frac{1}{\Lambda} H_{1}\left(L \cdot \varphi_{\tau}\right) \tau^{c}+\frac{1}{\Lambda^{2}} \theta H_{1}\left(L . \varphi_{\mu}\right) \mu^{c}+\frac{1}{\Lambda^{3}} \theta^{2} H_{1}\left(L \cdot \varphi_{e}\right) e^{c}
\end{aligned}
$$

where only these terms are allowed by the charges in table 2, where $L$ are the three families of electroweak lepton doublets unified into a single triplet of $S_{4}, \nu_{\mathrm{atm}}^{c}$ and $\nu_{\mathrm{sol}}^{c}$ are the (CP conjugated) right-handed neutrinos which are singlets of $S_{4}$, as are the two Higgs

\footnotetext{
${ }^{3}$ It is trivial to convert this superpotential to a non-supersymmetric Lagrangian by interpreting the leptons as fermions and the Higgs and flavons as scalars, rather than superfields. In the non-supersymmetric case it is possible to identify $H_{2}$ as the CP conjugate of the Higgs doublet $H_{1}$. It is also possible to absorb this Higgs doublet into the flavon fields so that $\phi_{i}$ and $\varphi_{\alpha}$ represent five $S_{4}$ triplets of Higgs doublets, corresponding to 15 Higgs doublets, in which case one power of $\Lambda$ would be removed from the denominator each term.
} 


\begin{tabular}{|c|c|c|c|c|c|c|c|c|c|c|c|c|c|c|}
\hline & $\theta \quad e^{c} \quad \mu^{c}$ & $\tau^{c} \varphi_{e}$ & $\varphi_{\mu}$ & $\varphi_{1}$ & & 1 & & & batm & $\phi_{\text {sol }}$ & $\nu_{\text {atm }}^{c}$ & & & \\
\hline$S_{4}$ & $\begin{array}{lll}1 & 1 & 1\end{array}$ & 13 & 3 & 3 & 1 & 3 & & 1 & $3^{\prime}$ & 3 & $\mathbf{1}^{\prime}$ & 1 & & 1 \\
\hline$Z_{3}$ & $1 \omega \omega^{2}$ & $1 \omega^{2}$ & $\omega$ & 1 & 1 & 1 & & 1 & $\omega$ & $\omega^{2}$ & $\omega^{2}$ & $\omega$ & & $\omega$ \\
\hline$Z_{3}^{\prime}$ & $\omega \omega^{2} \omega$ & $1 \omega^{2}$ & $\omega$ & 1 & 1 & 1 & & 1 & $\omega^{2}$ & 1 & $\omega$ & 1 & & 1 \\
\hline$R$ & $\begin{array}{ll}0 & 1\end{array}$ & 0 & 0 & 0 & 0 & 1 & & 0 & 0 & 0 & 1 & & 0 & 0 \\
\hline
\end{tabular}

Table 2. Lepton, Higgs and flavon superfields and how they may transform under the symmetries relevant for the Yukawa sector of the model. The $\mathrm{U}(1)_{R}$ symmetry, under which all the leptons have a charge of unity while the Higgs and flavons have zero charge, are also shown in the table.

doublets $H_{1,2}$, while $\phi_{i}$ and $\varphi_{\alpha}$ are $S_{4}$ triplet scalar flavons with the vacuum alignments in eqs. (13.1) and (13.2). Note that, according to the arguments of the previous section, all flavons are in the $\mathbf{3}$ representation apart from the atmospheric flavon which must be in the $\mathbf{3}^{\prime}$ which implies that the atmospheric right-handed neutrino must be in the $\mathbf{1}^{\prime}$ of $S_{4}$. We have introduced Majoron singlets $\xi_{i}$ whose VEVs will generate the diagonal righthanded neutrino masses. We have suppressed all dimensionless Yukawa coupling constants, assumed to be of order unity, with the charged lepton mass hierarchy originating from powers of $\theta$ which is an $S_{4}$ singlet. The corresponding powers of the mass scale $\Lambda$ keeps track of the mass dimension of each term.

Inserting the vacuum alignments in eqs. (13.1) and (13.2) into eqs. (14.1) and (14.2), we obtain the neutrino and charged lepton Yukawa matrices, with the rigid CSD(3) structure,

$$
Y^{\nu}=\left(\begin{array}{cc}
0 & y_{\text {sol }} \\
y_{\text {atm }} & 3 y_{\text {sol }} \\
y_{\text {atm }} & y_{\text {sol }}
\end{array}\right), \quad Y^{E}=\left(\begin{array}{ccc}
y_{e} & 0 & 0 \\
0 & y_{\mu} & 0 \\
0 & 0 & y_{\tau}
\end{array}\right),
$$

where

$$
y_{\text {atm }} \sim \frac{v_{\text {atm }}}{\Lambda}, \quad y_{\text {sol }} \sim \frac{v_{\text {sol }}}{\Lambda}, \quad y_{\tau} \sim \frac{v_{\tau}}{\Lambda}, \quad y_{\mu} \sim \frac{v_{\mu}\langle\theta\rangle}{\Lambda^{2}}, y_{e} \sim \frac{v_{e}\langle\theta\rangle^{2}}{\Lambda^{3}} .
$$

Note that we have a qualitative understanding of the charged lepton mass hierarchy as being due to successive powers of $\theta$, but there is no predictive power (for charged lepton masses) due to the arbitrary flavon VEVs and undetermined order unity dimensionless Yukawa couplings which we suppress.

When the Majorons get VEVs, the last two terms in eq. (14.1) will lead to the diagonal heavy Majorana mass matrix,

$$
M_{R}=\left(\begin{array}{cc}
M_{\mathrm{atm}} & 0 \\
0 & M_{\mathrm{sol}}
\end{array}\right),
$$

where $M_{\text {atm }} \sim\left\langle\xi_{\text {atm }}\right\rangle$ and $M_{\text {sol }} \sim\left\langle\xi_{\text {sol }}\right\rangle$. With the above seesaw matrices, we now have all the ingredients to reproduce the $\operatorname{CSD}(3)$ benchmark neutrino mass matrix in eq. (7.1), apart from the origin of the phase $\eta=2 \pi / 3$, which arises from vacuum alignment as we discuss in the next section. 


\section{Vacuum alignment in the $S_{4} \times Z_{3} \times Z_{3}^{\prime}$ model}

We have argued in section 13 that in general the vacuum the alignments in eqs. (13.1) and (13.2), arise as a consequence of $S_{4}$ residual symmetry, summarised by the starfish diagram in figure 2 , together with simple orthogonality conditions. It remains to show how this can be accomplished, together with the Majoron VEVs, by explicit superpotential alignment terms.

The charged lepton alignments in eq. (13.2) which naturally arise as a consequence of $S_{4}$, can be generated from the simple terms,

$$
W_{S_{4}}^{\mathrm{flav}, \ell}=A_{e} \varphi_{e} \varphi_{e}+A_{\mu} \varphi_{\mu} \varphi_{\mu}+A_{\tau} \varphi_{\tau} \varphi_{\tau} .
$$

where $A_{l}$ are $S_{4}$ triplet 3 driving fields with necessary $Z_{3} \times Z_{3}^{\prime}$ charges to absorb the charges of $\phi_{l}$ so as to allow the terms in eq. (15.1). F-flatness then leads to the desired charged lepton flavon alignments in eq. (13.2) due to,

$$
\left(\begin{array}{l}
\left\langle\phi_{l}\right\rangle_{2}\left\langle\phi_{l}\right\rangle_{3} \\
\left\langle\phi_{l}\right\rangle_{3}\left\langle\phi_{l}\right\rangle_{1} \\
\left\langle\phi_{l}\right\rangle_{1}\left\langle\phi_{l}\right\rangle_{2}
\end{array}\right)=\left(\begin{array}{l}
0 \\
0 \\
0
\end{array}\right)
$$

for $l=e, \mu, \tau$.

The vacuum alignment of the neutrino flavons involves the additional tribimaximal flavons $\phi_{i}$ with the orthogonality terms in eq. (5.3),

$$
W_{S_{4}}^{\mathrm{flav}, \text { perp }}=O_{i j} \phi_{i} \phi_{j}+O_{\mathrm{sol}} \phi_{\mathrm{sol}} \phi_{1}
$$

where we desire the tribimaximal alignments in eq. (13.3) and as usual we identify $\phi_{\text {atm }} \equiv$ $\phi_{3}$. We shall assume $\mathrm{CP}$ conservation with all triplet flavons acquiring real $\mathrm{CP}$ conserving VEVs. Since there is some freedom in the choice of $\phi_{1,2}$ charges under $Z_{3} \times Z_{3}^{\prime}$, we leave them unspecified. The singlet driving fields $O_{i j}$ and $O_{\text {sol }}$ have $R=2$ and $Z_{3} \times Z_{3}^{\prime}$ charges fixed by the (unspecified) $\phi_{i}$ charges,

The tribimaximal alignment for $\phi_{2}$ in the $\mathbf{3}$ in eq. (13.3) naturally arises as a consequence of $S_{4}$ from the simple terms,

$$
W_{S_{4}}^{\text {flav, TB2 }}=A_{2}\left(g_{2} \phi_{2} \phi_{2}+g_{2}^{\prime} \phi_{2} \xi_{2}\right) .
$$

where $A_{2}$ is an $R=2, S_{4}$ triplet $\mathbf{3}$ driving field and $\xi_{2}$ is a singlet, with the same (unspecified) $Z_{3} \times Z_{3}^{\prime}$ charge as $\phi_{2}$. F-flatness leads to,

$$
2 g_{2}\left(\begin{array}{c}
\left\langle\phi_{2}\right\rangle_{2}\left\langle\phi_{2}\right\rangle_{3} \\
\left\langle\phi_{2}\right\rangle_{3}\left\langle\phi_{2}\right\rangle_{1} \\
\left\langle\phi_{2}\right\rangle_{1}\left\langle\phi_{2}\right\rangle_{2}
\end{array}\right)+g_{2}^{\prime}\left\langle\xi_{2}\right\rangle\left(\begin{array}{c}
\left\langle\phi_{2}\right\rangle_{1} \\
\left\langle\phi_{2}\right\rangle_{2} \\
\left\langle\phi_{2}\right\rangle_{3}
\end{array}\right)=\left(\begin{array}{l}
0 \\
0 \\
0
\end{array}\right),
$$

leading to the tribimaximal alignment for $\phi_{2}$ in eq. (13.3). Note that in general the alignment derived from these $F$-term conditions is $\left\langle\phi_{2}\right\rangle \propto( \pm 1, \pm 1, \pm 1)^{T}$. These are all equivalent. For example $(1,1,-1)$ is related to permutations of the minus sign by $S_{4}$ transformations. The other choices can be obtained from these by simply multiplying an overall phase which would also change the sign of the $\xi_{2} \mathrm{VEV}$. 
The tribimaximal alignment for $\phi_{\mathrm{atm}} \equiv \phi_{3}$ in the $\mathbf{3}^{\prime}$ in eq. (13.3) naturally arises from

$$
W_{S_{4}}^{\text {flav,atm }}=A_{3}\left(g_{3} \phi_{3} \phi_{3}+g_{3}^{\prime} \phi_{e} \xi_{3}\right),
$$

where $A_{3}$ is an $S_{4}$ triplet 3 driving field and $\xi_{3}$ is a singlet, with suitable $Z_{3} \times Z_{3}^{\theta}$ charges assigned to all the fields so as to allow only these terms. F-flatness leads to,

$$
2 g_{3}\left(\begin{array}{c}
\left\langle\phi_{3}\right\rangle_{2}\left\langle\phi_{3}\right\rangle_{3} \\
\left\langle\phi_{3}\right\rangle_{3}\left\langle\phi_{3}\right\rangle_{1} \\
\left\langle\phi_{3}\right\rangle_{1}\left\langle\phi_{3}\right\rangle_{2}
\end{array}\right)+g_{3}^{\prime}\left\langle\xi_{2}\right\rangle\left(\begin{array}{c}
\left\langle\phi_{e}\right\rangle_{1} \\
\left\langle\phi_{e}\right\rangle_{2} \\
\left\langle\phi_{e}\right\rangle_{3}
\end{array}\right)=\left(\begin{array}{l}
0 \\
0 \\
0
\end{array}\right)
$$

which, using the orthogonality of $\phi_{2}$ and $\phi_{3}$ using eq. (15.3) and the pre-aligned electron flavon in eq. (13.2), leads to the tribimaximal alignment for $\phi_{\text {atm }} \equiv \phi_{3}$ in eq. (13.3). The tribimaximal alignment for $\phi_{1}$ then follows directly from the orthogonality conditions resulting from eq. (15.3).

The solar flavon alignment comes from the terms,

$$
W_{S_{4}}^{\mathrm{flav}, \mathrm{sol}}=A_{\mathrm{sol}}\left(g_{\mathrm{sol}} \phi_{\mathrm{sol}} \phi_{\mathrm{sol}}+g_{\mathrm{sol}}^{\prime} \phi_{\mathrm{sol}} \xi_{\mathrm{sol}}^{\prime}+g_{\mu} \phi_{\mu} \xi_{\mu}\right)
$$

F-flatness leads to,

$$
2 g_{\mathrm{sol}}\left(\begin{array}{c}
\left\langle\phi_{\mathrm{sol}}\right\rangle_{2}\left\langle\phi_{\mathrm{sol}}\right\rangle_{3} \\
\left\langle\phi_{\mathrm{sol}}\right\rangle_{3}\left\langle\phi_{\mathrm{sol}}\right\rangle_{1} \\
\left\langle\phi_{\mathrm{sol}}\right\rangle_{1}\left\langle\phi_{\mathrm{sol}}\right\rangle_{2}
\end{array}\right)+g_{\mathrm{sol}}^{\prime}\left\langle\xi_{\mathrm{sol}}^{\prime}\right\rangle\left(\begin{array}{c}
\left\langle\phi_{\mathrm{sol}}\right\rangle_{1} \\
\left\langle\phi_{\mathrm{sol}}\right\rangle_{2} \\
\left\langle\phi_{\mathrm{sol}}\right\rangle_{3}
\end{array}\right)+g_{\mu}\left\langle\xi_{\mu}\right\rangle\left(\begin{array}{c}
\left\langle\phi_{\mu}\right\rangle_{1} \\
\left\langle\phi_{\mu}\right\rangle_{2} \\
\left\langle\phi_{\mu}\right\rangle_{3}
\end{array}\right)=\left(\begin{array}{l}
0 \\
0 \\
0
\end{array}\right),
$$

which, using the pre-aligned muon flavon in eq. (13.2), leads to the form $(1, m, 1)$ for $\left\langle\phi_{\text {sol }}\right\rangle$, with $m$ unspecified, depending on the muon flavon VEV. On the other hand the last term in eq. (15.3) gives the general $\operatorname{CSD}(\mathrm{n})$ form in eq. (5.2), $(1, n, n-2)$ for $\left\langle\phi_{\text {sol }}\right\rangle$. The two constrained forms $(1, n, n-2)$ and $(1, m, 1)$, taken together, imply the unique alignment $(1,3,1)$ for $\phi_{\text {sol }}$ in eq. (13.1).

To understand the origin of the phase $\eta=2 \pi / 3$ we shall start by imposing exact CP invariance on the high energy theory, in eqs. (14.1) and (14.2), then spontaneously break $\mathrm{CP}$ in a very particular way, governed by the $Z_{3}$ symmetry, so that $\eta$ is restricted to be a cube root of unity. The Majoron flavon VEVs are driven by the superpotential,

$$
W_{S_{4}}^{\mathrm{flav}, \mathrm{maj}}=P\left(\frac{\xi_{\mathrm{atm}}^{3}}{\Lambda}-M^{2}\right)+P^{\prime}\left(\frac{\xi_{\text {sol }}^{3}}{\Lambda^{\prime}}-M^{\prime 2}\right)
$$

where $P, P^{\prime}$ are two copies of "driving" superfields with $R=2$ but transforming as singlets under all other symmetries, and $M$ is real due to CP conservation. Due to F-flatness,

$$
\left|\frac{\left\langle\xi_{\text {atm }}\right\rangle^{3}}{\Lambda}-M^{2}\right|^{2}=\left|\frac{\left\langle\xi_{\text {sol }}\right\rangle^{3}}{\Lambda^{\prime}}-M^{\prime 2}\right|^{2}=0 .
$$

These are satisfied by $\left\langle\xi_{\text {atm }}\right\rangle=\left|\left(\Lambda M^{2}\right)^{1 / 3}\right|$ and $\left\langle\xi_{\text {sol }}\right\rangle=\left|\left(\Lambda^{\prime} M^{\prime 2}\right)^{1 / 3}\right| e^{-2 i \pi / 3}$ where we arbitrarily select the phases to be zero and $-2 \pi / 3$ from amongst a discrete set of possible choices in each case. More generally we require a phase difference of $2 \pi / 3$ since the overall phase is not physically relevant, which would happen one in three times by chance. In the basis where the right-handed neutrino masses are real and positive this is equivalent to $\eta=2 \pi / 3$ in eq. (6.5), as in the benchmark model in eq. (7.1), due to the see-saw mechanism. 


\section{Conclusion}

The seesaw mechanism provides an elegant explanation of the smallness of neutrino masses. However in general it is difficult to test the mechanism experimentally, since the righthanded Majorana masses may have very large masses out of reach of high energy colliders. The heavy Majorana sector also introduces a new flavour sector, with yet more parameters, beyond those describing low energy neutrino physics. This is of serious concern, since the seesaw mechanism may be our best bet for extending the Standard Model to include neutrino masses.

Given that the seesaw mechanism is an elegant but practically untestable mechanism with a large number of parameters, in this paper we have relied on theoretical desiderata such as naturalness, minimality and predictability to guide us towards what we call the "Littlest Seesaw" model which is essentially the two right-handed neutrino model bundled together with further assumptions about the structure of the Yukawa couplings that we call $\operatorname{CSD}(n)$. Understandably one should be wary of such assumptions, indeed such principles of naturalness and minimality without experimental guidance could well prove to be unreliable. However we are encouraged by the fact that such principles in the guise of sequential dominance with a single texture zero, led to the bound $\theta_{13} \lesssim m_{2} / m_{3}$, suggesting a large reactor angle a decade before it was measured. The additional $\operatorname{CSD}(n)$ assumptions discussed here are simply designed to explain why this bound is saturated.

It is worth recapping the basic idea of sequential dominance that one of the righthanded neutrinos is dominantly responsible for the atmospheric neutrino mass, while a subdominant right-handed neutrino accounts for the solar neutrino mass, with possibly a third right-handed neutrino being approximately decoupled, leading to an effective two right-handed neutrino model. This simple idea leads to equally simple predictions which makes the scheme falsifiable. Indeed, the litmus test of such sequential dominance is Majorana neutrinos with a normal neutrino mass hiearchy and a very light (or massless) neutrino. These predictions will be tested soon.

In order to understand why the reactor angle bound is approximately saturated, we need to make additional assumptions, as mentioned. Ironically, the starting point is the original idea of constrained sequential dominance (CSD) which proved to be a good explanation of the tri-bimaximal solar and atmospheric angles but predicted a zero reactor angle. However, this idea can be generalised to the "Littlest Seesaw" comprising a two right-handed neutrino model with constrained Yukawa couplings of a particular $\operatorname{CSD}(n)$ structure, where here $n>1$ is taken to be a real parameter. We have shown that the reactor angle is given by $\theta_{13} \sim(n-1) \frac{\sqrt{2}}{3} \frac{m_{2}}{m_{3}}$ so that $n=1$ coresponds to original CSD with $\theta_{13}=0$, while $n=3$ corresponds to $\operatorname{CSD}(3)$ with $\theta_{13} \sim 2 \frac{\sqrt{2}}{3} \frac{m_{2}}{m_{3}}$, corresponding to $\theta_{13} \sim m_{2} / m_{3}$, which provides an explanation for why the SD bound is saturated as observed for this case, with both the approximation and SD breaking down for large $n$.

In general, the Littlest Seesaw is able to give a successful desciption of neutrino mass and the PMNS matrix in terms of four input parameters appearing in eq. (6.5) where the reactor angle requires $n \approx 3$. It predicts a normally ordered and very hierarchical neutrino mass spectrum with the lightest neutrino mass being zero. It also predicts TM1 
mixing with the atmospheric sum rules providing further tests of the scheme. Interestingly the single input phase $\eta$ must be responsible for $\mathcal{C P}$ violation in both neutrino oscillations and leptogenesis, providing the most direct link possible between these two phenomena. Indeed $\eta$ is identified as the leptogenesis phase. Another input parameter is $m_{b}$ which is identied with the neutrinoless double beta decay observable $m_{e e}$, although this is practically impossible to measure for $m_{1}=0$.

The main conceptual achievement in this paper is to realise that making $n$ continuous greatly simplifies the task of motivating the $\operatorname{CSD}(n)$ pattern of couplings, which emerge almost as simply as the TB couplings, as explained in figure 1. The main technical achievement of the paper is to provide exact analytic formulae for the lepton mixing angles, neutrino masses and CP phases in terms of the four input parameters of $\operatorname{CSD}(n)$ for any real $n>1$. The exact analytic results should facilitate phenomenological studies of the LS model. We have checked our analytic results against the numerical bechmark and validated them within the numerical precision. We also provided new simple analytic approximations such as: $\theta_{13} \sim(n-1) \frac{\sqrt{2}}{3} \frac{m_{2}}{m_{3}}$ where $m_{3} \approx 2 m_{a}$ and $m_{2} \approx 3 m_{b}$. The main model building achievement is to realise that the successful benchmark LS model based on $\operatorname{CSD}(3)$ is quite well motivated by a discrete $S_{4}$ symmetry, since the neutrino vacuum alignment directions are enforced by residual symmetries that are contained in $S_{4}$, but not $A_{4}$, which has hitherto been widely used in $\operatorname{CSD}(n)$ models. This is illustrated by the starfish diagram in figure 2 . In order to also fix the input leptogenesis phase to its benchmark value $\eta=2 \pi / 3$, we proposed a benchmark model, including supersymmetric vacuum alignment, based on $S_{4} \times Z_{3} \times Z_{3}^{\prime}$, which represents the simplest predictive seesaw model in the literature. The resulting benchmark predictions are: solar angle $\theta_{12}=34^{\circ}$, reactor angle $\theta_{13}=8.7^{\circ}$, atmospheric angle $\theta_{23}=46^{\circ}$, and Dirac phase $\delta_{\mathrm{CP}}=-87^{\circ}$. These predictions are all within the scope of future neutrino facilities, and may provide a useful target for them to aim at.

\section{Acknowledgments}

SFK acknowledges partial support from the STFC Consolidated ST/J000396/1 grant and the European Union FP7 ITN-INVISIBLES (Marie Curie Actions, PITN-GA-2011289442).

\section{A Lepton mixing conventions}

In the convention where the effective Lagrangian is given by ${ }^{4}$

$$
\mathcal{L}=-\overline{E_{L}} m^{E} E_{R}-\frac{1}{2} \overline{\nu_{L}} m^{\nu} \nu_{L}^{c}+H . c .
$$

Performing the transformation from the flavour basis to the real positive mass basis by,

$$
V_{E_{L}} m^{E} V_{E_{R}}^{\dagger}=m_{\text {diag }}^{E}=\operatorname{diag}\left(m_{e}, m_{\mu}, m_{\tau}\right), \quad V_{\nu_{L}} m^{\nu} V_{\nu_{L}}^{T}=m_{\text {diag }}^{\nu}=\operatorname{diag}\left(m_{1}, m_{2}, m_{3}\right),
$$

\footnotetext{
${ }^{4}$ Note that this convention for the light effective Majorana neutrino mass matrix $m^{\nu}$ differs by an overall complex conjugation compared to some other conventions in the literature.
} 
the PMNS matrix is given by

$$
U=V_{E_{L}} V_{\nu_{L}}^{\dagger}
$$

Since we are in the basis where the charged lepton mass matrix $m^{E}$ is already diagonal, then in general $V_{E_{L}}$ can only be a diagonal matrix,

$$
V_{E_{L}}=P_{E}=\left(\begin{array}{ccc}
e^{i \phi_{e}} & 0 & 0 \\
0 & e^{i \phi_{\mu}} & 0 \\
0 & 0 & e^{i \phi_{\tau}}
\end{array}\right)
$$

consisting of arbitrary phases, where an identical phase rotation on the right-handed charged leptons $V_{E_{R}}=P_{E}$ leaves the diagonal charged lepton masses in $m^{E}$ unchanged. In practice the phases in $P_{E}$ are chosen to absorb three phases from the unitary matrix $V_{\nu_{L}}^{\dagger}$ and to put $U$ in a standard convention [47],

$$
U=V P
$$

where, analogous to the CKM matrix,

$$
V=\left(\begin{array}{ccc}
c_{12} c_{13} & s_{12} c_{13} & s_{13} e^{-i \delta} \\
-s_{12} c_{23}-c_{12} s_{13} s_{23} e^{i \delta} & c_{12} c_{23}-s_{12} s_{13} s_{23} e^{i \delta} & c_{13} s_{23} \\
s_{12} s_{23}-c_{12} s_{13} c_{23} e^{i \delta} & -c_{12} s_{23}-s_{12} s_{13} c_{23} e^{i \delta} & c_{13} c_{23}
\end{array}\right)
$$

and the Majorana phase matrix factor is,

$$
P=\left(\begin{array}{ccc}
e^{i \frac{\beta_{1}}{2}} & 0 & 0 \\
0 & e^{i \frac{\beta_{2}}{2}} & 0 \\
0 & 0 & 1
\end{array}\right)
$$

From eqs. (A.2), (A.3), (A.4), we find,

$$
U^{\dagger} P_{E} m^{\nu} P_{E} U^{*}=\operatorname{diag}\left(m_{1}, m_{2}, m_{3}\right) .
$$

\section{B $\quad S_{4}$}

The irreducible representations of $S_{4}$ are two singlets $\mathbf{1}$ and $\mathbf{1}^{\prime}$, one doublet $\mathbf{2}$ and two triplets $\mathbf{3}$ and $\mathbf{3}^{\prime}$ [48]. The triplet $\mathbf{3}$ in the basis of [48] corresponds to the following 24 matrices,

$$
\begin{aligned}
a_{1}=\left(\begin{array}{lll}
1 & 0 & 0 \\
0 & 1 & 0 \\
0 & 0 & 1
\end{array}\right), & a_{2}=\left(\begin{array}{ccc}
1 & 0 & 0 \\
0 & -1 & 0 \\
0 & 0 & -1
\end{array}\right), & a_{3}=\left(\begin{array}{ccc}
-1 & 0 & 0 \\
0 & 1 & 0 \\
0 & 0 & -1
\end{array}\right), & a_{4}=\left(\begin{array}{ccc}
-1 & 0 & 0 \\
0 & -1 & 0 \\
0 & 0 & 1
\end{array}\right) \\
b_{1}=\left(\begin{array}{lll}
0 & 0 & 1 \\
1 & 0 & 0 \\
0 & 1 & 0
\end{array}\right), & b_{2}=\left(\begin{array}{ccc}
0 & 0 & 1 \\
-1 & 0 & 0 \\
0 & -1 & 0
\end{array}\right), & b_{3}=\left(\begin{array}{ccc}
0 & 0 & -1 \\
1 & 0 & 0 \\
0 & -1 & 0
\end{array}\right), & b_{4}=\left(\begin{array}{ccc}
0 & 0 & -1 \\
-1 & 0 & 0 \\
0 & 1 & 0
\end{array}\right) \\
c_{1}=\left(\begin{array}{lll}
0 & 1 & 0 \\
0 & 0 & 1 \\
1 & 0 & 0
\end{array}\right), & c_{2}=\left(\begin{array}{ccc}
0 & -1 & 0 \\
0 & 0 & -1 \\
-1 & 0 & 0
\end{array}\right), & c_{3}=\left(\begin{array}{ccc}
0 & 0 & 1 \\
-1 & 0 & 0
\end{array}\right), & c_{4}=\left(\begin{array}{ccc}
0 & 0 & -1 \\
1 & 0 & 0
\end{array}\right)
\end{aligned}
$$




$$
\begin{aligned}
& d_{1}=\left(\begin{array}{lll}
1 & 0 & 0 \\
0 & 0 & 1 \\
0 & 1 & 0
\end{array}\right), \quad d_{2}=\left(\begin{array}{ccc}
1 & 0 & 0 \\
0 & 0 & -1 \\
0 & -1 & 0
\end{array}\right), \quad d_{3}=\left(\begin{array}{ccc}
-1 & 0 & 0 \\
0 & 0 & 1 \\
0 & -1 & 0
\end{array}\right), \quad d_{4}=\left(\begin{array}{ccc}
-1 & 0 & 0 \\
0 & 0 & -1 \\
0 & 1 & 0
\end{array}\right) \\
& e_{1}=\left(\begin{array}{lll}
0 & 1 & 0 \\
1 & 0 & 0 \\
0 & 0 & 1
\end{array}\right), \quad e_{2}=\left(\begin{array}{ccc}
0 & 1 & 0 \\
-1 & 0 & 0 \\
0 & 0 & -1
\end{array}\right), \quad e_{3}=\left(\begin{array}{ccc}
0 & -1 & 0 \\
1 & 0 & 0 \\
0 & 0 & -1
\end{array}\right), \quad e_{4}=\left(\begin{array}{ccc}
0 & -1 & 0 \\
-1 & 0 & 0 \\
0 & 0 & 1
\end{array}\right) \\
& f_{1}=\left(\begin{array}{lll}
0 & 0 & 1 \\
0 & 1 & 0 \\
1 & 0 & 0
\end{array}\right), \quad f_{2}=\left(\begin{array}{ccc}
0 & 0 & 1 \\
0 & -1 & 0 \\
-1 & 0 & 0
\end{array}\right), \quad f_{3}=\left(\begin{array}{ccc}
0 & 0 & -1 \\
0 & 1 & 0 \\
-1 & 0 & 0
\end{array}\right), \quad f_{4}=\left(\begin{array}{ccc}
0 & 0 & -1 \\
0 & -1 & 0 \\
1 & 0 & 0
\end{array}\right)
\end{aligned}
$$

where $a_{i}, b_{i}, c_{i}$ are the 12 matrices of the $A_{4}$ triplet representation, while the remaining 12 matrices $d_{i}, e_{i}, f_{i}$ are the extra matrices in $S_{4}$. The triplet $\mathbf{3}^{\prime}$ in the basis of [48] corresponds to matrices which are are simply related to those above,

$$
\begin{array}{llll}
a_{1}^{\prime}=a_{1}, & a_{2}^{\prime}=a_{2}, & a_{3}^{\prime}=a_{3}, & a_{4}^{\prime}=a_{4}, \\
b_{1}^{\prime}=b_{1}, & b_{2}^{\prime}=b_{2}, & b_{3}^{\prime}=b_{3}, & b_{4}^{\prime}=b_{4}, \\
c_{1}^{\prime}=c_{1}, & c_{2}^{\prime}=c_{2}, & c_{3}^{\prime}=c_{3}, & c_{4}^{\prime}=c_{4}, \\
d_{1}^{\prime}=-d_{1}, & d_{2}^{\prime}=-d_{2}, & d_{3}^{\prime}=-d_{3}, & d_{4}^{\prime}=-d_{4}, \\
e_{1}^{\prime}=-e_{1}, & e_{2}^{\prime}=-e_{2}, & e_{3}^{\prime}=-e_{3}, & e_{4}^{\prime}=-e_{4}, \\
f_{1}^{\prime}=-f_{1}, & f_{2}^{\prime}=-f_{2}, & f_{3}^{\prime}=-f_{3}, & f_{4}^{\prime}=-f_{4} .
\end{array}
$$

In other words, for the $\mathbf{3}^{\prime}$, the 12 matrices $a_{i}^{\prime}, b_{i}^{\prime}, c_{i}^{\prime}$ associated with those of $A_{4}$ do not change sign, while the remaining 12 matrices $d_{i}^{\prime}, e_{i}^{\prime}, f_{i}^{\prime}$ involve a change of sign relative to the .

The Kronecker products of $S_{4}$ are: $\mathbf{1} \times \mathbf{1}=\mathbf{1}, \quad \mathbf{1}^{\prime} \times \mathbf{1}^{\prime}=\mathbf{1}, \quad \mathbf{1}^{\prime} \times \mathbf{1}=\mathbf{1}^{\prime}, \mathbf{3} \times \mathbf{3}=$ $1+2+3+3^{\prime}, \quad 3^{\prime} \times 3^{\prime}=1+2+3+3^{\prime}, \quad 3 \times 3^{\prime}=1^{\prime}+2+3+3^{\prime}, \quad 2 \times 2=1+1^{\prime}+2$, $\mathbf{2} \times \mathbf{3}=\mathbf{3}+\mathbf{3}^{\prime}, \quad \mathbf{2} \times \mathbf{3}^{\prime}=\mathbf{3}+\mathbf{3}^{\prime}$. The Clebsch relations in this basis are given in [48].

Open Access. This article is distributed under the terms of the Creative Commons Attribution License (CC-BY 4.0), which permits any use, distribution and reproduction in any medium, provided the original author(s) and source are credited.

\section{References}

[1] S.F. King, Models of Neutrino Mass, Mixing and CP-violation, J. Phys. G 42 (2015) 123001 [arXiv: 1510.02091] [INSPIRE].

[2] S.F. King, A. Merle, S. Morisi, Y. Shimizu and M. Tanimoto, Neutrino Mass and Mixing: from Theory to Experiment, New J. Phys. 16 (2014) 045018 [arXiv:1402.4271] [InSPIRE].

[3] S.F. King and C. Luhn, Neutrino Mass and Mixing with Discrete Symmetry, Rept. Prog. Phys. 76 (2013) 056201 [arXiv: 1301.1340] [INSPIRE].

[4] S.F. King, Neutrino mass models, Rept. Prog. Phys. 67 (2004) 107 [hep-ph/0310204] [INSPIRE]. 
[5] P. Minkowski, $\mu \rightarrow$ er at a Rate of One Out of $10^{9}$ Muon Decays?, Phys. Lett. B 67 (1977) 421 [INSPIRE].

[6] M. Gell-Mann, P. Ramond and R. Slansky, The Family Group in Grand Unified Theories, talk at Sanibel Symposium, Palm Coast U.S.A. (1979), CALT-68-709, and in Supergravity, North Holland, Amsterdam The Netherlands (1979).

[7] T. Yanagida, Horizontal Symmetry And Masses Of Neutrinos, in Proceedings of the Workshop on Unified Theory and Baryon Number of the Universe, KEK, Tsukuba Japan (1979)

[8] S.L. Glashow, in Cargese Lectures, Corsica France (1979).

[9] R.N. Mohapatra and G. Senjanović, Neutrino Mass and Spontaneous Parity Violation, Phys. Rev. Lett. 44 (1980) 912 [INSPIRE].

[10] J. Schechter and J.W.F. Valle, Neutrino Masses in $\mathrm{SU}(2) \times \mathrm{U}(1)$ Theories, Phys. Rev. D 22 (1980) 2227 [INSPIRE].

[11] S.F. King, Neutrino Mass and Mixing in the Seesaw Playground, arXiv:1511.03831 [INSPIRE].

[12] S.F. King, Atmospheric and solar neutrinos with a heavy singlet, Phys. Lett. B 439 (1998) 350 [hep-ph/9806440] [INSPIRE].

[13] S.F. King, Atmospheric and solar neutrinos from single right-handed neutrino dominance and U(1) family symmetry, Nucl. Phys. B 562 (1999) 57 [hep-ph/9904210] [InSPIRE].

[14] S.F. King, Large mixing angle $M S W$ and atmospheric neutrinos from single right-handed neutrino dominance and U(1) family symmetry, Nucl. Phys. B 576 (2000) 85 [hep-ph/9912492] [INSPIRE].

[15] S.F. King, Constructing the large mixing angle MNS matrix in seesaw models with right-handed neutrino dominance, JHEP 09 (2002) 011 [hep-ph/0204360] [INSPIRE].

[16] S.F. King, Leptogenesis MNS link in unified models with natural neutrino mass hierarchy, Phys. Rev. D 67 (2003) 113010 [hep-ph/0211228] [INSPIRE].

[17] P.F. Harrison, D.H. Perkins and W.G. Scott, Tri-bimaximal mixing and the neutrino oscillation data, Phys. Lett. B 530 (2002) 167 [hep-ph/0202074] [INSPIRE].

[18] S.F. King, Predicting neutrino parameters from $\mathrm{SO}(3)$ family symmetry and quark-lepton unification, JHEP 08 (2005) 105 [hep-ph/0506297] [INSPIRE].

[19] S. Antusch, S.F. King, C. Luhn and M. Spinrath, Trimaximal mixing with predicted $\theta_{13}$ from a new type of constrained sequential dominance, Nucl. Phys. B 856 (2012) 328 [arXiv: 1108.4278] [INSPIRE].

[20] S.F. King, Minimal predictive see-saw model with normal neutrino mass hierarchy, JHEP 07 (2013) 137 [arXiv: 1304.6264] [INSPIRE].

[21] S.F. King, Minimal see-saw model predicting best fit lepton mixing angles, Phys. Lett. B 724 (2013) 92 [arXiv:1305.4846] [INSPIRE].

[22] S.F. King, A model of quark and lepton mixing, JHEP 01 (2014) 119 [arXiv:1311.3295] [INSPIRE].

[23] S.F. King, A to $Z$ of Flavour with Pati-Salam, JHEP 08 (2014) 130 [arXiv:1406.7005] [INSPIRE]. 
[24] F. Björkeroth and S.F. King, Testing constrained sequential dominance models of neutrinos, J. Phys. G 42 (2015) 125002 [arXiv:1412.6996] [InSPIRE].

[25] F. Björkeroth, F.J. de Anda, I. de Medeiros Varzielas and S.F. King, Towards a complete $A_{4} \times \mathrm{SU}(5) S U S Y$ GUT, JHEP 06 (2015) 141 [arXiv:1503.03306] [INSPIRE].

[26] F. Björkeroth, F.J. de Anda, I. de Medeiros Varzielas and S.F. King, Leptogenesis in minimal predictive seesaw models, JHEP 10 (2015) 104 [arXiv:1505.05504] [INSPIRE].

[27] F. Björkeroth, F.J. de Anda, I.d.M. Varzielas and S.F. King, Towards a complete $\Delta(27) \times \mathrm{SO}(10) S U S Y$ GUT, arXiv:1512.00850 [INSPIRE].

[28] P.H. Frampton, S.L. Glashow and T. Yanagida, Cosmological sign of neutrino CP-violation, Phys. Lett. B 548 (2002) 119 [hep-ph/0208157] [INSPIRE].

[29] K. Harigaya, M. Ibe and T.T. Yanagida, Seesaw Mechanism with Occam's Razor, Phys. Rev. D 86 (2012) 013002 [arXiv:1205.2198] [INSPIRE].

[30] Z.-z. Xing and S. Zhou, Tri-bimaximal Neutrino Mixing and Flavor-dependent Resonant Leptogenesis, Phys. Lett. B 653 (2007) 278 [hep-ph/0607302] [INSPIRE].

[31] C.H. Albright and W. Rodejohann, Comparing Trimaximal Mixing and Its Variants with Deviations from Tri-bimaximal Mixing, Eur. Phys. J. C 62 (2009) 599 [arXiv:0812.0436] [INSPIRE].

[32] C.H. Albright, A. Dueck and W. Rodejohann, Possible Alternatives to Tri-bimaximal Mixing, Eur. Phys. J. C 70 (2010) 1099 [arXiv: 1004.2798] [InSPIRE].

[33] S.F. King and C. Luhn, On the origin of neutrino flavour symmetry, JHEP 10 (2009) 093 [arXiv: 0908.1897] [INSPIRE].

[34] C. Luhn, Trimaximal TM 1 neutrino mixing in $S_{4}$ with spontaneous CP-violation, Nucl. Phys. B 875 (2013) 80 [arXiv:1306.2358] [INSPIRE].

[35] M.-C. Chen and S.F. King, A4 See-Saw Models and Form Dominance, JHEP 06 (2009) 072 [arXiv: 0903.0125] [INSPIRE].

[36] S. Choubey, S.F. King and M. Mitra, On the Vanishing of the CP Asymmetry in Leptogenesis due to Form Dominance, Phys. Rev. D 82 (2010) 033002 [arXiv: 1004.3756] [INSPIRE].

[37] S.F. King, Vacuum misalignment corrections to tri-bimaximal mixing and form dominance, JHEP 01 (2011) 115 [arXiv: 1011.6167] [INSPIRE].

[38] M.C. Gonzalez-Garcia, M. Maltoni and T. Schwetz, Updated fit to three neutrino mixing: status of leptonic CP-violation, JHEP 11 (2014) 052 [arXiv:1409.5439] [INSPIRE].

[39] F. Capozzi, G.L. Fogli, E. Lisi, A. Marrone, D. Montanino and A. Palazzo, Status of three-neutrino oscillation parameters, circa 2013, Phys. Rev. D 89 (2014) 093018 [arXiv: 1312.2878] [INSPIRE].

[40] D.V. Forero, M. Tortola and J.W.F. Valle, Neutrino oscillations refitted, Phys. Rev. D 90 (2014) 093006 [arXiv: 1405.7540] [INSPIRE].

[41] C. Jarlskog, Commutator of the Quark Mass Matrices in the Standard Electroweak Model and a Measure of Maximal CP-violation, Phys. Rev. Lett. 55 (1985) 1039 [INSPIRE].

[42] J. Bernabeu, G.C. Branco and M. Gronau, CP restrictions on quark mass matrices, Phys. Lett. B 169 (1986) 243 [INSPIRE]. 
[43] G. Castelo-Branco and D. Emmanuel-Costa, Flavour Physics and CP-violation in the Standard Model and Beyond, Springer Proc. Phys. 161 (2015) 145 [arXiv:1402.4068] [INSPIRE].

[44] G.C. Branco, L. Lavoura and M.N. Rebelo, Majorana Neutrinos and CP Violation in the Leptonic Sector, Phys. Lett. B 180 (1986) 264 [INSPIRE].

[45] P. Ballett, S.F. King, C. Luhn, S. Pascoli and M.A. Schmidt, Testing atmospheric mixing sum rules at precision neutrino facilities, Phys. Rev. D 89 (2014) 016016 [arXiv:1308.4314] [INSPIRE].

[46] P. Ballett, S.F. King, C. Luhn, S. Pascoli and M.A. Schmidt, Precision measurements of $\theta_{12}$ for testing models of discrete leptonic flavour symmetries, J. Phys. Conf. Ser. 598 (2015) 012014 [arXiv: 1406.0308 ] [INSPIRE].

[47] Particle Data Group collaboration, K.A. Olive et al., Review of Particle Physics, Chin. Phys. C 38 (2014) 090001 [INSPIRE].

[48] H. Ishimori, T. Kobayashi, H. Ohki, Y. Shimizu, H. Okada and M. Tanimoto, Non-Abelian Discrete Symmetries in Particle Physics, Prog. Theor. Phys. Suppl. 183 (2010) 1 [arXiv: 1003.3552] [INSPIRE]. 